\title{
UNIFORM REGULARITY OF SET-VALUED MAPPINGS AND STABILITY OF IMPLICIT MULTIFUNCTIONS
}

\author{
Nguyen Duy Cuong * $\quad$ Alexander Y. Kruger ${ }^{\dagger}$
}

\begin{abstract}
We propose a unifying general (i.e. not assuming the mapping to have any particular structure) view on the theory of regularity and clarify the relationships between the existing primal and dual quantitative sufficient and necessary conditions including their hierarchy. We expose the typical sequence of regularity assertions, often hidden in the proofs, and the roles of the assumptions involved in the assertions, in particular, on the underlying space: general metric, normed, Banach or Asplund. As a consequence, we formulate primal and dual conditions for the stability properties of solution mappings to inclusions.
\end{abstract}

\section{INTRODUCTION}

Many important problems in variational analysis and optimization can be modelled by an inclusion $y \in F(x)$, where $F$ is a set-valued mapping. The behavior of the solution set $F^{-1}(y)$ when $y$ and/or $F$ are perturbed is of special interest. The concepts of metric regularity and subregularity (cf., e.g., [22,33, 46]) have been the key tools when studying stability of solutions. In the next definition, we use the names $\alpha$-regularity and $\alpha$-subregularity, fixing the main quantitative parameter in the conventional definitions of the properties.

Definition 1.1. Let $X$ and $Y$ be metric spaces, $F: X \rightrightarrows Y,(\bar{x}, \bar{y}) \in \operatorname{gph} F$, and $\alpha>0$. The mapping $F$ is

(i) $\alpha$-regular at $(\bar{x}, \bar{y})$ if there exist $\delta \in] 0,+\infty]$ and $\mu \in] 0,+\infty]$ such that

$$
\alpha d\left(x, F^{-1}(y)\right) \leq d(y, F(x))
$$

for all $x \in B_{\delta}(\bar{x})$ and $y \in B_{\delta}(\bar{y})$ with $d(y, F(x))<\alpha \mu$;

(ii) $\alpha$-subregular at $(\bar{x}, \bar{y})$ if there exist $\delta \in] 0,+\infty]$ and $\mu \in] 0,+\infty]$ such that

$$
\alpha d\left(x, F^{-1}(\bar{y})\right) \leq d(\bar{y}, F(x))
$$

for all $x \in B_{\delta}(\bar{x})$ with $d(\bar{y}, F(x))<\alpha \mu$.

The research was supported by the Australian Research Council, project DP160100854. The second author benefited from the support of the European Union's Horizon 2020 research and innovation programme under the Marie Skłodowska-Curie Grant Agreement No. 823731 CONMECH, and Conicyt REDES program 180032.

The authors thank the referees for their careful reading of the manuscript and constructive comments and suggestions.

* Centre for Informatics and Applied Optimization, School of Engineering, IT and Physical Sciences, Federation University, POB 663, Ballarat, Vic, 3350, Australia; Department of Mathematics, College of Natural Sciences, Can Tho University, Vietnam (duynguyen@students.federation.edu.au,ndcuong@ctu.edu.vn)

† Centre for Informatics and Applied Optimization, School of Engineering, IT and Physical Sciences, Federation University, POB 663, Ballarat, Vic, 3350, Australia (a.kruger@federation.edu.au, oRCID: 00oo-0002-7861-7380) 
In the above definition and throughout the paper, $B_{\delta}(\bar{x})$ and $B_{\delta}(\bar{y})$ stand for open balls with radius $\delta$ around respective points in appropriate spaces. Note that $\delta$ and $\mu$ can take infinite values; thus, the definition covers local as well as global properties. This remark applies also to the subsequent definitions. The technical conditions $d(y, F(x))<\alpha \mu$ and $d(\bar{y}, F(x))<\alpha \mu$ can be dropped (cf. [30,45]), particularly because the value $\mu=+\infty$ is allowed. This does not affect the properties themselves, but can have an effect on the value of $\delta$.

Inequalities (1.1) and (1.2) provide linear estimates of the distance from $x$ to the solution set of the respective inclusion via the 'residual' $d(y, F(x))$ or $d(\bar{y}, F(x))$. As commented by Dontchev and Rockafellar [22, p.178] 'in applications, the residual is typically easy to compute or estimate, whereas finding a solution might be considerably more difficult'.

Besides their importance in studying stability of solutions to inclusions, regularity type estimates are involved in constraint qualifications for optimization problems, qualification conditions in subdifferential and coderivative calculus, and convergence analysis of computational algorithms $[1,3-5,12,16$, 23, 27, 43].

The name 'metric regularity' was coined by Borwein in 1986 [8], but the concept itself can be traced back to the Banach-Schauder open mapping theorem for linear operators, and its nonlinear extensions due to Lyusternik \& Graves [26,44] and Robinson \& Ursescu [53,59]; see, for instance, [19, 20, 22,31,45,57] for historical comments. Unlike the 'full' regularity in part (i) of Definition 1.1, the weaker subregularity property in part (ii) (as well as closely related to it properties like calmness, error bounds and weak sharp minima) is not stable under small perturbations of the data. It has also been well studied; see, for instance, $[2,10,20,22,38,42,45,61,63]$. Fortunately, the subregularity property is satisfied automatically in finite dimensions when the graph of $F$ is the union of finitely many polyhedral convex sets; cf. [22,33].

When $y$ is not fixed and can be any point in a neighbourhood of a given point $\bar{y}$, it represents canonical perturbations of the inclusion $\bar{y} \in F(x)$. For some applications it can be important to allow also perturbations in the right-hand side. This leads to the need to consider parametric inclusions $\bar{y} \in F(p, x)$ (or even $y \in F(p, x)$, thus, combining the two types of perturbations), where $F$ is a setvalued mapping of two variables, with (nonlinear) perturbations in the right-hand side given by a parameter $p$ from some fixed set $P$.

Along with the mapping $F: P \times X \rightrightarrows Y$, which is our main object in this paper, given a point $p \in P$, we consider the mapping $F_{p}:=F(p, \cdot): X \rightrightarrows Y$. Given a $y \in Y$, the mapping

$$
p \mapsto G(p):=F_{p}^{-1}(y)=\{x \in X \mid y \in F(p, x)\}
$$

can be interpreted as an implicit multifunction corresponding to the parametric inclusion $y \in F(p, x)$. When studying implicit multifunctions, it is common to consider 'uniform' versions of the properties in Definition 1.1 (cf., e.g., [32, Definition 3.1]).

Definition 1.2. Let $X$ and $Y$ be metric spaces, and $P$ be a set, $F: P \times X \rightrightarrows Y, \bar{x} \in X, \bar{y} \in Y$, and $\alpha>0$. The mapping $F$ is

(i) $\alpha$-regular in $x$ uniformly in $p$ over $P$ at $(\bar{x}, \bar{y})$ if there exist $\delta \in] 0,+\infty]$ and $\mu \in] 0,+\infty]$ such that

$$
\alpha d\left(x, F_{p}^{-1}(y)\right) \leq d(y, F(p, x))
$$

for all $p \in P, x \in B_{\delta}(\bar{x})$ and $y \in B_{\delta}(\bar{y})$ with $d(y, F(p, x))<\alpha \mu$;

(ii) $\alpha$-subregular in $x$ uniformly in $p$ over $P$ at $(\bar{x}, \bar{y})$ if there exist $\delta \in] 0,+\infty]$ and $\mu \in] 0,+\infty]$ such that

$$
\alpha d\left(x, F_{p}^{-1}(\bar{y})\right) \leq d(\bar{y}, F(p, x))
$$

for all $p \in P$ and $x \in B_{\delta}(\bar{x})$ with $d(\bar{y}, F(p, x))<\alpha \mu$. 
If $P$ is a singleton, then the properties in Definition 1.2 reduce to the corresponding conventional regularity properties in Definition 1.1. Moreover, the subregularity property in Definition 1.2(ii) coincides in this case with the subregularity property of the mapping (1.3) considered in [14].

Remark 1.3. (i) If $Y$ is a linear metric space with a shift-invariant metric, in particular, a normed space, then the property in part (i) of Definition 1.2 reduces to the one in part (ii) with the extended parameter set $\widehat{P}:=P \times Y$ and set-valued mapping $\widehat{F}((p, y), x):=F(p, x)-y,((p, y), x) \in \widehat{P} \times X$, in place of $P$ and $F$, respectively. Moreover, in both parts of the definition, it is sufficient to consider the case $\bar{y}:=0$ : the general case reduces to it by replacing $F$ with $F-\bar{y}$.

(ii) Unlike Definition 1.1, in Definition 1.2 the reference point $(\bar{x}, \bar{y})$ is not associated with the graph of $F$. This is a technical relaxation caused by the fact that gph $F$ is a subset of a product of three spaces $P \times X \times Y$, and at this stage there is no reference point in $P$. Definition 1.4 below is formulated in a more conventional way.

(iii) There exist other concepts of uniform regularity in the literature. For instance, it is not uncommon to talk about uniform regularity when inequality (1.1) holds for all $(\bar{x}, \bar{y})$ in a compact subset of $X \times Y$ with the same parameters $\alpha, \delta$ and $\mu$; cf. [16].

Local (in $p$ ) versions of the properties in Definition 1.2 are of special interest. They correspond to $P$ being a neighbourhood of a point $\bar{p}$ in some metric spaces; cf., e.g., [32, 49].

Definition 1.4. Let $P, X$ and $Y$ be metric spaces, $F: P \times X \rightrightarrows Y,(\bar{p}, \bar{x}, \bar{y}) \in \operatorname{gph} F$, and $\alpha>0$. The mapping $F$ is

(i) $\alpha$-regular in $x$ uniformly in $p$ at $(\bar{p}, \bar{x}, \bar{y})$ if there exist $\eta \in] 0,+\infty], \delta \in] 0,+\infty]$ and $\mu \in] 0,+\infty]$ such that inequality (1.4) is satisfied for all $p \in B_{\eta}(\bar{p}), x \in B_{\delta}(\bar{x})$ and $y \in B_{\delta}(\bar{y})$ with $d(y, F(p, x))<$ $\alpha \mu$;

(ii) $\alpha$-subregular in $x$ uniformly in $p$ at $(\bar{p}, \bar{x}, \bar{y})$ if there exist $\eta \in] 0,+\infty], \delta \in] 0,+\infty]$ and $\mu \in] 0,+\infty]$ such that inequality (1.5) is satisfied for all $p \in B_{\eta}(\bar{p})$ and $x \in B_{\delta}(\bar{x})$ with $d(\bar{y}, F(p, x))<\alpha \mu$.

We often simply say that $F$ is regular or subregular if the exact value of $\alpha$ in the above definitions is not important. The exact upper bound of all $\alpha>0$ such that a property in the above definitions is satisfied with some $\delta \in] 0,+\infty]$ and $\mu \in] 0,+\infty]$ (and $\eta \in] 0,+\infty]$ ), is called the modulus (or rate) of the property.

Apart from the main parameter $\alpha$, providing a quantitative measure of the respective property, the properties in above definitions depend also on the auxiliary parameters $\delta, \eta$ and $\mu$. They control (directly and indirectly) the size of the neighbourhoods of $\bar{x}$ and $\bar{p}$ involved in the definitions. As discussed above, the last parameter can be dropped (together with the corresponding constraints). We keep all the parameters to emphasize their different roles in the definitions and corresponding characterizations. The necessary and sufficient regularity conditions presented in the paper normally involve the same collection of parameters.

The properties in Definitions 1.2 and 1.4 can be interpreted as kinds of Lipschitz-like properties of the implicit multifunction (solution mapping) (1.3). This observation opens a way for numerous applications of the characterizations established in this and many other papers; cf. Section 5 .

Regularity properties of implicit multifunctions were first considered by Robinson [52-54] when studying stability of solution sets of generalized equations. This initiated a great deal of research by many authors, mostly in normed spaces (and with $\bar{y}:=0$ ). Dontchev et al. [21, Theorem 2.1] gave a sufficient condition for regularity of implicit multifunctions in terms of graphical derivatives. Ngai et al. $[47,49]$ employed the theory of error bounds to characterizing the property in metric and Banach spaces. In $[14,15,25,28,29,40,41,50,60]$ dual sufficient conditions were established in finite and infinite dimensions in terms of Fréchet, limiting, directional limiting and Clarke coderivatives. Chieu et al. [11] established connections between regularity and Lipschitz-like properties of implicit multifunctions. 
The regularity properties of the type given in Definitions 1.2 and 1.4 are often referred to in the literature as metric regularity [14, 29, 41], metric regularity in Robinson's sense [50,60], and Robinson metric regularity [11,28] (of implicit multifunctions). Following Ioffe [32,33], we prefer to talk about uniform regularity. We refer the readers to $[6,7,22,32,40,50]$ for more discussions and historical comments.

The metric properties in Definition 1.4 admit equivalent geometric characterizations. This is illustrated by the next proposition providing a characterization for the property in Definition 1.2(ii).

Proposition 1.5. Let $X$ and $Y$ be metric spaces, and $P$ be a set, $F: P \times X \rightrightarrows Y, \bar{x} \in X, \bar{y} \in Y$, and $\alpha>0$. The mapping $F$ is $\alpha$-subregular in $x$ uniformly in $p$ over $P$ at $(\bar{x}, \bar{y})$ with some $\delta \in] 0,+\infty]$ and $\mu \in] 0,+\infty]$ if and only if

$$
F_{p}^{-1}(\bar{y}) \cap B_{\rho}(x) \neq \emptyset
$$

for all $\rho \in] 0, \mu\left[, p \in P\right.$ and $x \in B_{\delta}(\bar{x})$ with $d(\bar{y}, F(p, x))<\alpha \rho$.

Proof. Suppose $F$ is $\alpha$-subregular in $x$ uniformly in $p$ over $P$ at $(\bar{x}, \bar{y})$ with some $\delta \in] 0,+\infty]$ and $\mu \in] 0,+\infty]$. Let $\rho \in] 0, \mu\left[, p \in P\right.$ and $x \in B_{\delta}(\bar{x})$ with $d(\bar{y}, F(p, x))<\alpha \rho$. Then $d(\bar{y}, F(p, x))<\alpha \mu$. By Definition 1.2(ii), $d\left(x, F_{p}^{-1}(\bar{y})\right) \leq \alpha^{-1} d(\bar{y}, F(p, x))<\rho$. Hence, condition (1.6) is satisfied.

Conversely, suppose $\delta \in] 0,+\infty]$ and $\mu \in] 0,+\infty]$, and condition (1.6) is satisfied for all $\rho \in] 0, \mu[, p \in P$ and $x \in B_{\delta}(\bar{x})$ with $d(\bar{y}, F(p, x))<\alpha \rho$. Let $p \in P$ and $x \in B_{\delta}(\bar{x})$ with $d(\bar{y}, F(p, x))<\alpha \mu$. Choose a $\rho$ satisfying $\alpha^{-1} d(\bar{y}, F(p, x))<\rho<\mu$. Then, by (1.6), $d\left(x, F_{p}^{-1}(\bar{y})\right)<\rho$. Letting $\rho \downarrow \alpha^{-1} d(\bar{y}, F(p, x))$, we arrive at (1.5), i.e. $F$ is $\alpha$-subregular in $x$ uniformly in $p$ over $P$ at $(\bar{x}, \bar{y})$ with $\delta$ and $\mu$.

The aim of this paper is not to add some new sufficient or necessary conditions for regularity properties of general set-valued mappings or implicit multifunctions to the large volume of existing ones (although some conditions in the subsequent sections are indeed new), but to propose a unifying general (i.e. not assuming the mapping $F$ to have any particular structure and not using tangential approximations of gph $F$ ) view on the theory of regularity, and clarify the relationships between the existing conditions including their hierarchy. We expose the typical sequence of regularity assertions, often hidden in the proofs, and the roles of the assumptions involved in the assertions, in particular, on the underlying space: general metric, normed, Banach or Asplund.

We present a series of necessary and sufficient regularity conditions with the main emphasis (in line with the current trend in the literature) on the latter ones. The (typical) sequence of sufficient regularity conditions is represented by the following chain of assertions, each subsequent assertion being a consequence of the previous one:

(i) nonlocal primal space conditions in complete metric spaces (Theorem 3.1(ii));

(ii) local primal space conditions in complete metric spaces (Corollary 3.4(ii));

(iii) subdifferential conditions in Banach and Asplund spaces (Proposition 4.1);

(iv) normal cone conditions in Banach and Asplund spaces (Theorem 4.4);

(v) coderivative conditions in Banach and Asplund spaces (Corollaries 4.8 and 4.9).

Even if one targets coderivative conditions, they still have to go through the five steps listed above with details often hidden in long proofs. Apart from making the whole process more transparent, which is our main objective, the assertions in (i)-(iv) can be of independent interest, at least theoretically, especially the slope type conditions in (ii) and normal cone conditions in (iv). In combination with tangential approximations of gph $F$, they are likely to lead to verifiable regularity conditions.

The implications (i) $\Rightarrow$ (ii) and (iv) $\Rightarrow$ (v) in the above list follow immediately from the definitions. The main assertions are the sufficiency of condition (i), and implications (ii) $\Rightarrow$ (iii) $\Rightarrow$ (iv). They employ the following fundamental tools of variational analysis: 
- Ekeland variational principle (sufficiency of condition (i));

- sum rules for respective subdifferentials (implications (ii) $\Rightarrow$ (iii) $\Rightarrow$ (iv)).

Thus, all the sufficient conditions on the list are consequences of the Ekeland variational principle, and as such, they are 'outer' conditions, i.e. they need to be checked at points outside the solution set $F_{p}^{-1}(\bar{y})$.

Most of the sufficient conditions are accompanied by the corresponding necessary ones. The necessary conditions do not require the underlying spaces to be complete and are generally easy consequences of the definitions. With the exception of the general nonlocal condition in Theorem 3.1(i), such conditions are formulated in normed spaces and assume the graph of $F$ to be convex. In Section 4.2, we provide a series of dual necessary regularity conditions for set-valued mappings with closed convex graphs acting between Banach spaces some of which are also sufficient.

In the setting of complete metric spaces, and assuming that $g p h F_{p}$ is closed for all $p \in P$, the gap between the nonlocal necessary and sufficient subregularity conditions in Theorem 3.1 is not big: they share the same inequality (3.3); with all the other parameters coinciding, the sufficiency part naturally requires it to hold for all $x$ in a larger set. Unfortunately, unlike the 'full' regularity possessing the well known coderivative criterion (see, e.g., $[36,45]$ ), this is not the case in general with local subregularity conditions unless the graph of $F$ is convex. The sufficient subregularity conditions presented in the paper are the weakest possible in each group, but can still be far from necessary. As it has been discussed in the literature (see, e.g., a discussion of the equivalent subtransversality property in [39]), the reason for this phenomenon lies in the fact that the subregularity property lacks robustness.

The hot topic of regularity of a set-valued mapping $F$ with a special structure, particularly in the arising in numerous applications such as, e.g., KKT systems and variational inequalities, case when $F=g+G$ with $g$ single valued and $G$ set-valued (typically a normal cone mapping), is outside the scope of the current paper. Computing 'slopes' and coderivatives of such mappings (or normal cones to their graphs) is usually a difficult job and requires imposing additional assumptions on $g$ and $G$. This is what people working in this area normally do. We want to emphasize that this type of conditions still fall into the five-point scheme described above.

The rest of the paper is organized as follows. The next Section 2 contains some preliminary facts used throughout the paper. Sections 3 and 4 are dedicated, respectively, to primal and dual sufficient and necessary conditions for the regularity properties. In Section 5 , we illustrate the theory by characterizing the conventional metric regularity and subregularity of set-valued mappings as well as stability properties of solution mappings to parametric inclusions.

\section{PRELIMINARIES}

Our basic notation is standard, see, e.g., $[22,45,57]$. Throughout the paper, if not explicitly stated otherwise, $P$ is an arbitrary set, $X$ and $Y$ are either metric or normed/Banach/Asplund spaces. Products of metric or normed spaces are assumed to be equipped with the maximum distance or norm. The topological dual of a normed space $X$ is denoted by $X^{*}$, while $\langle\cdot, \cdot\rangle$ denotes the bilinear form defining the pairing between the two spaces. In a primal space, the open and closed balls with center $x$ and radius $\delta \in] 0,+\infty]$ are denoted, respectively, by $B_{\delta}(x)$ and $\bar{B}_{\delta}(x)$, while $\mathbb{B}$ and $\overline{\mathbb{B}}$ stand for, respectively, the open and closed unit balls. The open unit ball in the dual space is denoted by $\mathbb{B}^{*}$. Symbols $\mathbb{R}$, $\mathbb{R}_{+}$and $\mathbb{N}$ stand for the real line, the set of all nonnegative reals, and the set of all positive integers, respectively.

For a set $\Omega$ in a normed space, its closure is denoted by $\operatorname{cl} \Omega$. The distance from a point $x$ to $\Omega$ is defined by $d(x, \Omega):=\inf _{u \in \Omega}\|u-x\|$, and we use the convention $d(x, \emptyset)=+\infty$. The indicator function of $\Omega$ is defined by $i_{\Omega}(x)=0$ if $x \in \Omega$, and $i_{\Omega}(x)=+\infty$ if $x \notin \Omega$. 
The dual conditions in the paper are formulated in terms of Fréchet and Clarke normals and subdifferentials; cf., e.g., [17,37].

Given a point $\bar{x} \in \Omega$, the sets

$$
\begin{gathered}
N_{\Omega}^{F}(\bar{x}):=\left\{x^{*} \in X^{*} \mid \limsup _{\Omega \ni x \rightarrow \bar{x}, x \neq \bar{x}} \frac{\left\langle x^{*}, x-\bar{x}\right\rangle}{\|x-\bar{x}\|} \leq 0\right\}, \\
N_{\Omega}^{C}(\bar{x}):=\left\{x^{*} \in X^{*} \mid\left\langle x^{*}, z\right\rangle \leq 0 \text { for all } z \in T_{\Omega}^{C}(\bar{x})\right\}
\end{gathered}
$$

are, respectively, the Fréchet and Clarke normal cones to $\Omega$ at $\bar{x}$. In definition (2.2), $T_{\Omega}^{C}(\bar{x})$ stands for the Clarke tangent cone to $\Omega$ at $\bar{x}$. The sets (2.1) and (2.2) are nonempty closed convex cones satisfying $N_{\Omega}^{F}(\bar{x}) \subset N_{\Omega}^{C}(\bar{x})$. If $\Omega$ is a convex set, both cones reduce to the normal cone in the sense of convex analysis:

$$
N_{\Omega}(\bar{x}):=\left\{x^{*} \in X^{*} \mid\left\langle x^{*}, x-\bar{x}\right\rangle \leq 0 \text { for all } x \in \Omega\right\} .
$$

For an extended-real-valued function $f: X \rightarrow \mathbb{R} \cup\{+\infty\}$ on a normed space, its domain and epigraph are defined, respectively, by $\operatorname{dom} f:=\{x \in X \mid f(x)<+\infty\}$ and epi $f:=\{(x, \alpha) \in X \times \mathbb{R} \mid f(x) \leq \alpha\}$. The Fréchet and Clarke subdifferentials of $f$ at $\bar{x} \in \operatorname{dom} f$ are defined, respectively, as

$$
\begin{gathered}
\partial^{F} f(\bar{x}):=\left\{x^{*} \in X^{*} \mid \liminf _{x \rightarrow \bar{x}, x \neq \bar{x}} \frac{f(x)-f(\bar{x})-\left\langle x^{*}, x-\bar{x}\right\rangle}{\|x-\bar{x}\|} \geq 0\right\}, \\
\partial^{C} f(\bar{x}):=\left\{x^{*} \in X^{*} \mid\left\langle x^{*}, z\right\rangle \leq f^{\circ}(\bar{x}, z) \text { for all } z \in X\right\},
\end{gathered}
$$

where $f^{\circ}(\bar{x}, z)$ is the Clarke-Rockafellar directional derivative $[55,56]$ of $f$ at $\bar{x}$ in the direction $z \in X$. The sets (2.3) and (2.4) are closed and convex, and satisfy $\partial^{F} f(\bar{x}) \subset \partial^{C} f(\bar{x})$. If $f$ is convex, they reduce to the subdifferential in the sense of convex analysis:

$$
\partial f(\bar{x}):=\left\{x^{*} \in X^{*} \mid f(x)-f(\bar{x})-\left\langle x^{*}, x-\bar{x}\right\rangle \geq 0 \text { for all } x \in X\right\} .
$$

It is easy to check that $N_{\Omega}^{F}(\bar{x})=\partial^{F} i_{\Omega}(\bar{x}), N_{\Omega}^{C}(\bar{x})=\partial^{C} i_{\Omega}(\bar{x})$, and

$$
\begin{aligned}
& \partial^{F} f(\bar{x})=\left\{x^{*} \in X^{*} \mid\left(x^{*},-1\right) \in N_{\text {epi } f}^{F}(\bar{x}, f(\bar{x}))\right\}, \\
& \partial^{C} f(\bar{x})=\left\{x^{*} \in X^{*} \mid\left(x^{*},-1\right) \in N_{\text {epi } f}^{C}(\bar{x}, f(\bar{x}))\right\} .
\end{aligned}
$$

By convention, we set $N_{\Omega}^{F}(\bar{x})=N_{\Omega}^{C}(\bar{x}):=\emptyset$ if $\bar{x} \notin \Omega$ and $\partial^{F} f(\bar{x})=\partial^{C} f(\bar{x}):=\emptyset$ if $x \notin \operatorname{dom} f$. We often use the generic notations $N$ and $\partial$ for Fréchet and Clarke objects, specifying wherever necessary the type of the object by an appropriate superscript, e.g., $N:=N^{F}$ or $N:=N^{C}$.

The following fact is an immediate consequence of the definition of the Fréchet subdifferential; cf. [37,45].

Lemma 2.1. Suppose $X$ is a normed space and $f: X \rightarrow \mathbb{R} \cup\{+\infty\}$. If $\bar{x} \in \operatorname{dom} f$ is a point of local minimum of $f$, then $0 \in \partial^{F} f(\bar{x})$.

The representation of the (convex) subdifferential of a norm in the next lemma is of importance; cf. [62, Corollary 2.4.16].

Lemma 2.2. Let $(Y,\|\cdot\|)$ be a normed space. Then

(i) $\partial\|\cdot\|(0)=\left\{y^{*} \in Y^{*} \mid\left\|y^{*}\right\| \leq 1\right\}$;

(ii) $\partial\|\cdot\|(y)=\left\{y^{*} \in Y^{*} \mid\left\langle y^{*}, y\right\rangle=\|y\|\right.$ and $\left.\left\|y^{*}\right\|=1\right\}, \quad y \neq 0$. 
For an extended real-valued function $f$ on a metric space, its slope and nonlocal slope (cf. [7,30,38,48]) at $x \in \operatorname{dom} f$ are defined, respectively, by

$$
|\nabla f|(x):=\limsup _{u \rightarrow x, u \neq x} \frac{[f(x)-f(u)]_{+}}{d(x, u)} \text { and }|\nabla f|^{\diamond}(x):=\sup _{u \neq x} \frac{\left[f(x)-f_{+}(u)\right]_{+}}{d(x, u)},
$$

where $\alpha_{+}:=\max \{0, \alpha\}$ for any $\alpha \in \mathbb{R}$. If $x \notin \operatorname{dom} f$, we set $|\nabla f|(x)=|\nabla f|^{\diamond}(x):=+\infty$. The following simple facts are well known; cf., e.g., [18].

Lemma 2.3. Let $X$ be a metric space, $f: X \rightarrow \mathbb{R} \cup\{+\infty\}, x \in \operatorname{dom} f$, and $f(x)>0$.

(i) $|\nabla f|(x) \leq|\nabla f|^{\diamond}(x)$.

(ii) If $X$ is a normed space and $f$ is convex, then $|\nabla f|^{\diamond}(x)=|\nabla f|(x)=d(0, \partial f(x))$.

A set-valued mapping $F: X \rightrightarrows Y$ between two sets $X$ and $Y$ is a mapping, which assigns to every $x \in X$ a subset (possibly empty) $F(x)$ of $Y$. We use the notations gph $F:=\{(x, y) \in X \times Y \mid y \in F(x)\}$ and dom $F:=\{x \in X \mid F(x) \neq \emptyset\}$ for the graph and the domain of $F$, respectively, and $F^{-1}: Y \rightrightarrows X$ for the inverse of $F$. This inverse (which always exists with possibly empty values at some $y$ ) is defined by $F^{-1}(y):=\{x \in X \mid y \in F(x)\}, y \in Y$. Obviously $\operatorname{dom} F^{-1}=F(X)$.

If $X$ and $Y$ are normed spaces, the coderivative of $F$ at $(x, y) \in \operatorname{gph} F$ is a set-valued mapping $D^{*} F(x, y): Y^{*} \rightrightarrows X^{*}$ defined by

$$
D^{*} F(x, y)\left(y^{*}\right):=\left\{x^{*} \in X^{*} \mid\left(x^{*},-y^{*}\right) \in N_{\operatorname{gph} F}(x, y)\right\}, \quad y^{*} \in Y^{*} .
$$

Depending on the type of the normal cone in (2.5), it can define various coderivatives. We use symbols $D_{F}^{*}$ and $D_{C}^{*}$ to denote, respectively, the Fréchet and Clarke coderivatives.

The key tools in the proofs of the main results are the celebrated Ekeland variational principle and several subdifferential sum rules; cf. [22, 24, 33, 34, 37, 45, 55, 62].

Lemma 2.4. Suppose $X$ is a complete metric space, $f: X \rightarrow \mathbb{R} \cup\{+\infty\}$ is lower semicontinuous, $x \in X$, $\varepsilon>0$ and $\lambda>0$. If $f(x)<\inf _{X} f+\varepsilon$, then there exists an $\hat{x} \in X$ such that

(i) $d(\hat{x}, x)<\lambda$;

(ii) $f(\hat{x}) \leq f(x)$;

(iii) $f(u)+(\varepsilon / \lambda) d(u, \hat{x}) \geq f(\hat{x})$ for all $u \in X$.

Lemma 2.5. Suppose $X$ is a normed space, $f_{1}, f_{2}: X \rightarrow \mathbb{R} \cup\{+\infty\}$, and $\bar{x} \in \operatorname{dom} f_{1} \cap \operatorname{dom} f_{2}$.

(i) Let $f_{1}$ and $f_{2}$ be convex and $f_{1}$ be continuous at a point in dom $f_{2}$. Then

$$
\partial\left(f_{1}+f_{2}\right)(\bar{x})=\partial f_{1}(\bar{x})+\partial f_{2}(\bar{x}) .
$$

(ii) Let $f_{1}$ be Lipschitz continuous and $f_{2}$ be lower semicontinuous in a neighbourhood of $\bar{x}$. Then

$$
\partial^{C}\left(f_{1}+f_{2}\right)(\bar{x}) \subset \partial^{C} f_{1}(\bar{x})+\partial^{C} f_{2}(\bar{x}) .
$$

(iii) Let $X$ be Asplund, $f_{1}$ be Lipschitz continuous and $f_{2}$ be lower semicontinuous in a neighbourhood of $\bar{x}$. Then, for any $x^{*} \in \partial^{F}\left(f_{1}+f_{2}\right)(\bar{x})$ and $\varepsilon>0$, there exist $x_{1}, x_{2} \in X$ with $\left\|x_{i}-\bar{x}\right\|<\varepsilon$, $\left|f_{i}\left(x_{i}\right)-f_{i}(\bar{x})\right|<\varepsilon(i=1,2)$, such that

$$
x^{*} \in \partial^{F} f_{1}\left(x_{1}\right)+\partial^{F} f_{2}\left(x_{2}\right)+\varepsilon \mathbb{B}^{*} .
$$

Recall that a Banach space is Asplund if every continuous convex function on an open convex set is Fréchet differentiable on a dense subset [51], or equivalently, if the dual of each its separable subspace is separable. We refer the reader to $[9,45,51]$ for discussions about and characterizations of Asplund spaces. All reflexive, particularly, all finite dimensional Banach spaces are Asplund. 


\section{SLOPE NECESSARY AND SUFFICIENT CONDITIONS}

This section is dedicated to slope necessary and sufficient conditions. For simplicity, we focus on the uniform subregularity property in Definition 1.2(ii). The corresponding conditions for the property in Definition 1.2(i) can be formulated in a similar way. Besides, in view of Remark 1.3, in normed spaces (which is our setting in the next section) such conditions can be obtained as consequences of those for the subregularity.

The necessary conditions are deduced directly from the definitions of the respective properties, while the sufficient ones come from the application of the Ekeland variational principle. In the convex case, the conditions are necessary and sufficient.

In this section, $P$ is a nonempty set, $X$ and $Y$ are metric spaces, and $F: P \times X \rightrightarrows Y$. We assume the parameters $\bar{x} \in X, \bar{y} \in Y, \alpha>0, \delta \in] 0,+\infty]$ and $\mu \in] 0,+\infty]$ to be fixed. In what follows, we employ a collection of functions

$$
\psi_{p}(u, v):=d(v, \bar{y})+i_{\operatorname{gph} F_{p}}(u, v), \quad u \in X, v \in Y
$$

depending on a parameter $p \in P$. Along with the standard maximum distance on $X \times Y$, we also use a metric depending on a parameter $\gamma>0$ :

$$
d_{\gamma}((u, v),(x, y)):=\max \{d(u, x), \gamma d(v, y)\}, \quad u, x \in X, v, y \in Y .
$$

The next theorem plays a crucial role for the subsequent considerations. The slope and subdifferential/normal cone/coderivative conditions for uniform $\alpha$-subregularity in this paper are consequences of this theorem.

Theorem 3.1. (i) If $F$ is $\alpha$-subregular in $x$ uniformly in $p$ over $P$ at $(\bar{x}, \bar{y})$ with $\delta$ and $\mu$, then

$$
\sup _{\substack{(u, v) \in g p h F_{p},(u, v) \neq(x, y) \\ d(u, \bar{x})<\delta+\mu, d(v, \bar{y})<\alpha \mu}} \frac{d(y, \bar{y})-d(v, \bar{y})}{d_{\gamma}((u, v),(x, y))} \geq \alpha
$$

for $\gamma:=\alpha^{-1}$, and all $p \in P, x \in B_{\delta}(\bar{x})$ and $y \in Y$ satisfying

$$
x \notin F_{p}^{-1}(\bar{y}), \quad y \in F(p, x) \cap B_{\alpha \mu}(\bar{y}) .
$$

(ii) Suppose $X$ and $Y$ are complete, and $g p h F_{p}$ is closed for all $p \in P$. If inequality (3.3) holds for some $\gamma>0$, and all $p \in P, x \in B_{\delta+\mu}(\bar{x})$ and $y \in Y$ satisfying (3.4), then $F$ is $\alpha$-subregular in $x$ uniformly in $p$ over $P$ at $(\bar{x}, \bar{y})$ with $\delta$ and $\mu$.

Proof. (i) Suppose $F$ is $\alpha$-subregular in $x$ uniformly in $p$ over $P$ at $(\bar{x}, \bar{y})$ with $\delta$ and $\mu$. Let $p \in P$, $x \in B_{\delta}(\bar{x})$ and $y \in Y$ satisfy (3.4), $\gamma:=\alpha^{-1}$, and $\eta>1$. By (3.4) and Definition 1.2(ii), there exist a $\xi \in] 1, \eta\left[\right.$ such that $\xi d(y, \bar{y})<\alpha \mu$, and a point $\hat{x} \in F_{p}^{-1}(\bar{y})$ such that $\alpha d(x, \hat{x})<\xi d(y, \bar{y})$. Thus, $(\hat{x}, \bar{y}) \in \operatorname{gph} F_{p},(\hat{x}, \bar{y}) \neq(x, y)$,

$$
\begin{gathered}
d(\hat{x}, \bar{x}) \leq d(\hat{x}, x)+d(x, \bar{x})<\alpha^{-1} \xi d(y, \bar{y})+\delta \leq \mu+\delta, \quad \text { and } \\
d_{y}((x, y),(\hat{x}, \bar{y}))=\max \{d(x, \hat{x}), \gamma d(y, \bar{y})\} \leq \alpha^{-1} \max \{\xi, 1\} d(y, \bar{y})=\alpha^{-1} \xi d(y, \bar{y}) .
\end{gathered}
$$

Hence,

$$
\sup _{\substack{(u, v) \in \operatorname{gph} F_{p},(u, v) \neq(x, y) \\ d(u, \bar{x})<\delta+\mu, d(v, \bar{y})<\alpha \mu}} \frac{d(y, \bar{y})-d(v, \bar{y})}{d_{y}((u, v),(x, y))} \geq \frac{d(y, \bar{y})}{d_{\gamma}((\hat{x}, \bar{y}),(x, y))} \geq \alpha \xi^{-1}>\alpha \eta^{-1} .
$$

Letting $\eta \downarrow 1$, we arrive at (3.3). 
(ii) Suppose $F$ is not $\alpha$-subregular in $x$ uniformly in $p$ over $P$ at $(\bar{x}, \bar{y})$ with $\delta$ and $\mu$. By Definition 1.2(ii), there exist points $p \in P$ and $x \in B_{\delta}(\bar{x})$ such that

$$
d(\bar{y}, F(p, x))<\alpha \min \left\{d\left(x, F_{p}^{-1}(\bar{y})\right), \mu\right\} .
$$

Hence, $x \notin F_{p}^{-1}(\bar{y})$, or equivalently, $\bar{y} \notin F(p, x)$. Set $\mu_{0}:=\min \left\{d\left(x, F_{p}^{-1}(\bar{y})\right), \mu\right\}$. Choose a number $\varepsilon$ such that $d(\bar{y}, F(p, x))<\varepsilon<\alpha \mu_{0}$, and a point $y \in F(p, x)$ such that $d(y, \bar{y})<\varepsilon$. The function $\psi_{p}: X \times Y \rightarrow \mathbb{R}_{+} \cup\{+\infty\}$, defined by (3.1), is lower semicontinuous on $\bar{B}_{\delta+\mu}(\bar{x}) \times \bar{B}_{\alpha \mu}(\bar{y})$. Besides,

$$
\psi_{p}(x, y)=d(y, \bar{y})<\inf _{\bar{B}_{\delta+\mu}(\bar{x}) \times \bar{B}_{\alpha \mu}(\bar{y})} \psi_{p}+\varepsilon .
$$

Let $\gamma>0$. Applying the Ekeland variational principle (Lemma 2.4) to the restriction of $\psi_{p}$ to the complete metric space $\bar{B}_{\delta+\mu}(\bar{x}) \times \bar{B}_{\alpha \mu}(\bar{y})$ with the metric (3.2), we can find a point $(\hat{x}, \hat{y}) \in$ $\bar{B}_{\delta+\mu}(\bar{x}) \times \bar{B}_{\alpha \mu}(\bar{y})$ such that

$$
\begin{gathered}
d_{y}((\hat{x}, \hat{y}),(x, y))<\mu_{0}, \quad \psi_{p}(\hat{x}, \hat{y}) \leq \psi_{p}(x, y), \\
\psi_{p}(\hat{x}, \hat{y}) \leq \psi_{p}(u, v)+\left(\varepsilon / \mu_{0}\right) d_{y}((u, v),(\hat{x}, \hat{y}))
\end{gathered}
$$

for all $(u, v) \in \bar{B}_{\delta+\mu}(\bar{x}) \times \bar{B}_{\alpha \mu}(\bar{y})$. By (3.5), we have $\hat{y} \in F(p, \hat{x})$, and

$$
\begin{gathered}
d(\hat{x}, \bar{x}) \leq d(\hat{x}, x)+d(x, \bar{x})<\mu_{0}+\delta \leq \mu+\delta, \\
d(\hat{y}, \bar{y}) \leq d(y, \bar{y})<\varepsilon<\alpha \mu_{0} \leq \alpha \mu .
\end{gathered}
$$

Besides, $d(\hat{x}, x)<\mu_{0} \leq d\left(x, F_{p}^{-1}(\bar{y})\right)$. This implies $\hat{x} \notin F_{p}^{-1}(\bar{y})$, and consequently, $\hat{y} \neq \bar{y}$. It follows from (3.6) that

$$
\sup _{\substack{(u, v) \in \operatorname{gph} F_{p},(u, v) \neq(\hat{x}, \hat{y}) \\ d(u, \bar{x})<\delta+\mu, d(v, \bar{y})<\alpha \mu}} \frac{d(\hat{y}, \bar{y})-d(v, \bar{y})}{d_{\gamma}((u, v),(\hat{x}, \hat{y}))} \leq \frac{\varepsilon}{\mu_{0}}<\alpha .
$$

The last estimate contradicts (3.3).

Remark 3.2. (i) The expression in the left-hand side of the inequality (3.3) is the nonlocal $\gamma$-slope [38, p. 6o] at $(x, y)$ of the restriction of the function $\psi_{p}$, given by (3.1), to $\operatorname{gph} F_{p} \cap\left[B_{\delta+\mu}(\bar{x}) \times B_{\alpha \mu}(\bar{y})\right]$.

(ii) By the definition of the metric (3.2), if inequality (3.3) is satisfied with a $\gamma>0$, then it is also satisfied with any $\left.\gamma^{\prime} \in\right] 0, \gamma[$. This observation is applicable to all slope inequalities in this section.

(iii) The completeness of the space and closedness assumption in part (ii) of Theorem 3.1 (and the subsequent statements) can be relaxed: it suffices to require that $\operatorname{gph} F_{p} \cap\left[\bar{B}_{\delta+\mu}(\bar{x}) \times \bar{B}_{\alpha \mu}(\bar{y})\right]$ is complete for all $p \in P$.

(iv) The sufficient condition in part (ii) of Theorem 3.1 is often hidden in the proofs of dual sufficient conditions.

(v) When $X$ and $Y$ are complete, and gph $F_{p}$ is closed for all $p \in P$, the gap between the nonlocal necessary and sufficient regularity conditions in parts (i) and (ii) of Theorem 3.1 is not big: they share the same inequality (3.3); with all the other parameters coinciding, the necessity part (i) guarantees this inequality to hold for all $x \in B_{\delta}(\bar{x})$, while the sufficiency part (ii) requires it to hold for all $x$ in a larger set $B_{\delta+\mu}(\bar{x})$. 
We now illustrate Theorem 3.1 by applying it to the local (in $p$ ) setting in Definition 1.4(ii). The application is straightforward. We provide a single illustration of this kind, although the other statements in this and the next section are also applicable to this setting.

Corollary 3.3. Let $P$ be a metric space, $(\bar{p}, \bar{x}, \bar{y}) \in g p h F$ and $\eta \in] 0,+\infty]$.

(i) If $F$ is $\alpha$-subregular in $x$ uniformly in $p$ at $(\bar{p}, \bar{x}, \bar{y})$ with $\eta, \delta$ and $\mu$, then inequality (3.3) holds with $\gamma:=\alpha^{-1}$ for all $p \in B_{\eta}(\bar{p}), x \in B_{\delta}(\bar{x})$ and $y \in Y$ satisfying (3.4).

(ii) Suppose $X$ and $Y$ are complete, and $g p h F_{p}$ is closed for all $p \in B_{\eta}(\bar{p})$. If inequality (3.3) holds for some $\gamma>0$, and all $p \in B_{\eta}(\bar{p}), x \in B_{\delta+\mu}(\bar{x})$ and $y \in Y$ satisfying (3.4), then $F$ is $\alpha$-subregular in $x$ uniformly in $p$ at $(\bar{p}, \bar{x}, \bar{y})$ with $\eta, \delta$ and $\mu$.

The next statement presents a localized version of Theorem 3.1.

Corollary 3.4. (i) Suppose $X$ and $Y$ are normed spaces, and gph $F_{p}$ is convex for all $p \in P$. If $F$ is $\alpha$-subregular in $x$ uniformly in $p$ over $P$ at $(\bar{x}, \bar{y})$ with $\delta$ and $\mu$, then

$$
\limsup _{\substack{u \rightarrow x, v \rightarrow y,(u, v) \in g p h F_{p},(u, v) \neq(x, y) \\ d(u, \bar{x})<\delta+\mu, d(v, \bar{y})<\alpha \mu}} \frac{d(y, \bar{y})-d(v, \bar{y})}{d_{y}((u, v),(x, y))} \geq \alpha
$$

for $\gamma:=\alpha^{-1}$, and all $p \in P, x \in B_{\delta}(\bar{x})$ and $y \in Y$ satisfying (3.4).

(ii) Suppose $X$ and $Y$ are complete, and $g p h F_{p}$ is closed for all $p \in P$. If inequality (3.7) holds for some $\gamma>0$, and all $p \in P, x \in B_{\delta+\mu}(\bar{x})$ and $y \in Y$ satisfying (3.4), then $F$ is $\alpha$-subregular in $x$ uniformly in $p$ over $P$ at $(\bar{x}, \bar{y})$ with $\delta$ and $\mu$.

Proof. In view of Remark 3.2(i) and Remark 3.5(i), assertion (i) follows from Lemma 2.3(ii) and Theorem 3.1(i), while assertion (ii) is a consequence of Lemma 2.3(i) and Theorem 3.1(ii).

Remark 3.5. (i) The expression in the left-hand side of inequality (3.7) is the $\gamma$-slope [38, p. 61] at $(x, y)$ of the restriction of the function $\psi_{p}$, given by (3.1), to gph $F_{p} \cap\left[B_{\delta+\mu}(\bar{x}) \times B_{\alpha \mu}(\bar{y})\right]$.

(ii) The convexity assumption in part (i) of Corollary 3.4 (and the subsequent statements) can be relaxed: it suffices to require that $\mathrm{gph} F_{p} \cap\left[\bar{B}_{\delta+\mu}(\bar{x}) \times \bar{B}_{\alpha \mu}(\bar{y})\right]$ is convex for all $p \in P$.

(iii) In the particular case when $P$ is a neighborhood of a point $\bar{p}$ in some metric space, part (ii) of Corollary 3.4 is a quantitative version of [32, Proposition 3.5]. Ngai et al. [49, Theorem 3] established a primal sufficient condition for the property under the assumption that the mapping $F(\cdot, \bar{x})$ is lower semicontinuous at $\bar{p}$.

\section{DUAL NECESSARY AND SUFFICIENT CONDITIONS}

In this section, we continue studying the mapping $F: P \times X \rightrightarrows Y$ where $P$ is a nonempty set, while $X$ and $Y$ are assumed to be normed spaces. We also assume the parameters $\bar{x} \in X, \bar{y} \in Y, \alpha>0$, $\delta \in] 0,+\infty]$ and $\mu \in] 0,+\infty]$ to be fixed, and the collection of functions $\psi_{p}$ be defined by (3.1).

The primal and dual parametric product space norms, corresponding to the distance (3.2), have the following form:

$$
\begin{gathered}
\|(x, y)\|_{\gamma}=\max \{\|x\|, y\|y\|\}, \quad x \in X, \quad y \in Y, \\
\left\|\left(x^{*}, y^{*}\right)\right\|_{\gamma}=\left\|x^{*}\right\|+\gamma^{-1}\left\|y^{*}\right\|, \quad x^{*} \in X^{*}, y^{*} \in Y^{*} .
\end{gathered}
$$

We denote by $d_{\gamma}$ the distance in $X^{*} \times Y^{*}$ determined by (4.2). 


\subsection{DUAL SUFFICIENT CONDITIONS}

In this subsection, we assume additionally that $X$ and $Y$ are Banach spaces, and gph $F_{p}$ is closed for all $p \in P$.

The next subdifferential sufficient condition for uniform $\alpha$-subregularity is a consequence of Corollary 3.4(ii) thanks to the subdifferential sum rules in Lemma 2.5 .

Proposition 4.1. Let $\partial:=\partial^{C}$. If

$$
d_{\gamma}\left(0, \partial \psi_{p}(x, y)\right) \geq \alpha
$$

for some $\gamma>0$, and all $p \in P, x \in B_{\delta+\mu}(\bar{x})$ and $y \in Y$ satisfying (3.4), then $F$ is $\alpha-$ subregular in $x$ uniformly in $p$ over $P$ at $(\bar{x}, \bar{y})$ with $\delta$ and $\mu$.

If $X$ and $Y$ are Asplund, then the above assertion is valid with $\partial:=\partial^{F}$.

Proof. Suppose $F$ is not $\alpha$-subregular in $x$ uniformly in $p$ over $P$ at $(\bar{x}, \bar{y})$ with $\delta$ and $\mu$. Let $\gamma>0$. By Corollary 3.4(ii), there exist points $p \in P, x \in B_{\delta+\mu}(\bar{x})$ and $y \in Y$ satisfying (3.4), and an $\left.\alpha^{\prime} \in\right] 0, \alpha[$ such that

$$
\|y-\bar{y}\|-\|v-\bar{y}\| \leq \alpha^{\prime}\|(u, v)-(x, y)\|_{\gamma}
$$

for all $(u, v) \in \operatorname{gph} F_{p} \cap\left[B_{\delta+\mu}(\bar{x}) \times B_{\alpha \mu}(\bar{y})\right]$ near $(x, y)$. In other words, $(x, y)$ is a local minimizer of the function

$$
(u, v) \mapsto \psi_{p}(u, v)+\alpha^{\prime}\|(u, v)-(x, y)\|_{\gamma}
$$

By Lemma 2.1, its Fréchet and, as a consequence, Clarke subdifferential at this point contains 0. Observe that (4.4) is the sum of the function $\psi_{p}$ and the Lipschitz continuous convex function $(u, v) \mapsto \alpha^{\prime}\|(u, v)-(x, y)\|_{\gamma}$, and, by Lemma 2.2, at any point all subgradients $\left(x^{*}, y^{*}\right)$ of the latter function satisfy $\left\|\left(x^{*}, y^{*}\right)\right\|_{\gamma} \leq \alpha^{\prime}$. By Lemma 2.5(ii), there exists a subgradient $\left(x^{*}, y^{*}\right) \in \partial^{C} \psi_{p}(x, y)$ such that $\left\|\left(x^{*}, y^{*}\right)\right\|_{\gamma} \leq \alpha^{\prime}<\alpha$, which contradicts (4.3).

Let $X$ and $Y$ be Asplund. Choose an $\varepsilon>0$ such that

$$
\varepsilon<\min \left\{\delta+\mu-\|x-\bar{x}\|, \alpha \mu-\|y-\bar{y}\|, \alpha-\alpha^{\prime},\|y-\bar{y}\| / 2, d\left(x, F_{p}^{-1}(\bar{y})\right) / 2\right\}
$$

By Lemma 2.5(iii), there exist points $x^{\prime} \in B_{\varepsilon}(x), y^{\prime} \in B_{\varepsilon}(y)$ with $\left(x^{\prime}, y^{\prime}\right) \in$ gph $F_{p}$, and a subgradient $\left(x^{*}, y^{*}\right) \in \partial^{F} \psi_{p}\left(x^{\prime}, y^{\prime}\right)$ such that

$$
\left\|\left(x^{*}, y^{*}\right)\right\|_{\gamma}<\alpha^{\prime}+\varepsilon<\alpha .
$$

Besides, $x^{\prime} \in B_{\delta+\mu}(\bar{x}) \backslash F_{p}^{-1}(\bar{y}), \bar{y} \neq y^{\prime} \in B_{\alpha \mu}(\bar{y})$ as

$$
\begin{gathered}
\|y-\bar{y}\| / 2<\left\|y^{\prime}-\bar{y}\right\|, \quad d\left(x, F_{p}^{-1}(\bar{y})\right) / 2<d\left(x^{\prime}, F_{p}^{-1}(\bar{y})\right), \\
\left\|x^{\prime}-\bar{x}\right\| \leq\left\|x^{\prime}-x\right\|+\|x-\bar{x}\|<\delta+\mu, \quad\left\|y^{\prime}-\bar{y}\right\| \leq\left\|y^{\prime}-y\right\|+\|y-\bar{y}\|<\alpha \mu .
\end{gathered}
$$

It follows from $(4.5)$ that $d_{\gamma}\left(0, \partial^{F} \psi_{p}\left(x^{\prime}, y^{\prime}\right)\right)<\alpha$, which contradicts (4.3).

Remark 4.2. Condition (4.3) with the Fréchet subdifferentials is obviously weaker (hence, more efficient) than its version with the Clarke ones. However, it is only applicable in Asplund spaces.

The key condition (4.3) in Proposition 4.1 involves subdifferentials of the function $\psi_{p}$. Subgradients of this function belong to $X^{*} \times Y^{*}$ and have two component vectors $x^{*}$ and $y^{*}$. In view of the representation (4.2) of the dual norm on $X^{*} \times Y^{*}$, the contributions of the vectors $x^{*}$ and $y^{*}$ to the condition $(4.3)$ are different. The next corollary exposes this difference. 
Corollary 4.3. If there exists an $\varepsilon>0$ such that $\left\|x^{*}\right\| \geq \alpha$ for all $p \in P, x \in B_{\delta+\mu}(\bar{x})$ and $y \in Y$ satisfying (3.4), and all $\left(x^{*}, y^{*}\right) \in \partial^{C} \psi_{p}(x, y)$ with $\left\|y^{*}\right\|<\varepsilon$; particularly if

$$
\liminf _{\substack{F_{p}^{-1}(\bar{y}) \nexists x \rightarrow \bar{x}, F(p, x) \ni y \rightarrow \bar{y}, y^{*} \rightarrow 0 \\ p \in P, y \neq \bar{y},\left(x^{*}, y^{*}\right) \in \partial^{C} \psi_{p}(x, y)}}\left\|x^{*}\right\|>\alpha,
$$

then $F$ is $\alpha$-subregular in $x$ uniformly in $p$ over $P$ at $(\bar{x}, \bar{y})$ with $\delta$ and $\mu$.

If $X$ and $Y$ are Asplund, then the above assertion is valid with $\partial^{F}$ in place of $\partial^{C}$.

Proof. Suppose $F$ is not $\alpha$-subregular in $x$ uniformly in $p$ over $P$ at $(\bar{x}, \bar{y})$ with $\delta$ and $\mu$. Let $\varepsilon>0$ and $\gamma:=\varepsilon / \alpha$. By Proposition 4.1, there exist $p \in P, x \in B_{\delta+\mu}(\bar{x})$ and $y \in Y$ satisfying (3.4), and a subgradient $\left(x^{*}, y^{*}\right) \in \partial^{C} \psi_{p}(x, y)\left(\left(x^{*}, y^{*}\right) \in \partial^{F} \psi_{p}(x, y)\right.$ if $X$ and $Y$ are Asplund $)$ such that $\left\|\left(x^{*}, y^{*}\right)\right\|_{\gamma}<\alpha$. In view of the representation of the dual norm (4.2), this implies $\left\|x^{*}\right\|<\alpha$ and $\left\|y^{*}\right\|<\alpha \gamma=\varepsilon$, a contradiction.

The function $\psi_{p}$ involved in the subdifferential sufficient conditions for the uniform $\alpha$-subregularity in Proposition 4.1, is itself a sum of two functions. We are now going to apply the sum rules again to obtain sufficient conditions in terms of Clarke and Fréchet normals to gph $F_{p}$.

Theorem 4.4. The mapping $F$ is $\alpha$-subregular in $x$ uniformly in $p$ over $P$ at $(\bar{x}, \bar{y})$ with $\delta$ and $\mu$ if, for some $\gamma>0$, and all $p \in P, x \in B_{\delta+\mu}(\bar{x})$ and $y \in Y$ satisfying (3.4), one of the following conditions is satisfied:

(i) with $N:=N^{C}$,

$$
d_{y}\left(\left(0,-y^{*}\right), N_{g p h F_{p}}(x, y)\right) \geq \alpha
$$

for all $y^{*} \in Y^{*}$ satisfying

$$
\left\|y^{*}\right\|=1, \quad\left\langle y^{*}, y-\bar{y}\right\rangle=\|y-\bar{y}\|
$$

(ii) $X$ and $Y$ are Asplund, and there exists a $\tau \in] 0,1\left[\right.$ such that inequality (4.6) holds with $N:=N^{F}$ for all $y^{*} \in Y^{*}$ satisfying

$$
\left\|y^{*}\right\|=1, \quad\left\langle y^{*}, y-\bar{y}\right\rangle>\tau\|y-\bar{y}\| .
$$

Proof. Suppose $F$ is not $\alpha$-subregular in $x$ uniformly in $p$ over $P$ at $(\bar{x}, \bar{y})$ with $\delta$ and $\mu$. Let $\gamma>0$. In view of Proposition 4.1, there exist $p \in P, x \in B_{\delta+\mu}(\bar{x})$ and $y \in Y$ satisfying (3.4), and a subgradient $\left(\hat{x}^{*}, \hat{y}^{*}\right) \in \partial \psi_{p}(x, y)$ such that $\left\|\left(\hat{x}^{*}, \hat{y}^{*}\right)\right\|_{\gamma}<\alpha$, where either $\partial:=\partial^{C}$ (if $X$ and $Y$ are general Banach spaces) or $\partial:=\partial^{F}$ (if $X$ and $Y$ are Asplund). Recall from (3.1) that $\psi_{p}$ is a sum of two functions: the Lipschitz continuous convex function $v \mapsto g(v):=\|v-\bar{y}\|$ and the indicator function of the closed set gph $F_{p}$.

(i) By Lemma 2.5(ii), there exist $y^{*} \in \partial g(y)$ and $\left(u^{*}, v^{*}\right) \in N_{\operatorname{gph} F_{p}}^{C}(x, y)$ such that $\left(\hat{x}^{*}, \hat{y}^{*}\right)=$ $\left(0, y^{*}\right)+\left(u^{*}, v^{*}\right)$. Thus,

$$
d_{\gamma}\left(\left(0,-y^{*}\right), N_{\mathrm{gph} F_{p}}^{C}(x, y)\right) \leq\left\|\left(0, y^{*}\right)+\left(u^{*}, v^{*}\right)\right\|_{\gamma}=\left\|\left(\hat{x}^{*}, \hat{y}^{*}\right)\right\|_{\gamma}<\alpha,
$$

which contradicts (4.6). Since $y \neq \bar{y}$, by Lemma 2.2, $y^{*}$ satisfies conditions (4.7). 
(ii) Let $X$ and $Y$ be Asplund, and $\tau \in] 0,1\left[\right.$. By Lemma 2.5(iii), for any $\varepsilon>0$, there exist $x_{1} \in B_{\varepsilon}(x)$, $y_{1}, y_{2} \in B_{\varepsilon}(y)$ with $\left(x_{1}, y_{1}\right) \in \operatorname{gph} F_{p}$, and $y^{*} \in \partial g\left(y_{2}\right),\left(u^{*}, v^{*}\right) \in N_{\operatorname{gph} F_{p}}^{F}\left(x_{1}, y_{1}\right)$ such that

$$
\left\|\left(0, y^{*}\right)+\left(u^{*}, v^{*}\right)-\left(\hat{x}^{*}, \hat{y}^{*}\right)\right\|_{\gamma}<\varepsilon .
$$

The number $\varepsilon$ can be chosen small enough to ensure that $x_{1} \in B_{\delta+\mu}(\bar{x}) \backslash F_{p}^{-1}(\bar{y}), y_{1} \in B_{\alpha \mu}(\bar{y})$, $y_{2} \neq \bar{y}$, and

$$
\left\|y_{1}-\bar{y}\right\| \geq \frac{1}{2}\|y-\bar{y}\|,\left\|y_{2}-y_{1}\right\|<\frac{1-\tau}{4}\|y-\bar{y}\|,\left\|\left(\hat{x}^{*}, \hat{y}^{*}\right)\right\|_{\gamma}+\varepsilon<\alpha .
$$

By Lemma 2.2, we have $\left\|y^{*}\right\|=1$ and $\left\langle y^{*}, y_{2}-\bar{y}\right\rangle=\left\|y_{2}-\bar{y}\right\|$. Moreover,

$$
\left\|y_{2}-y_{1}\right\|<\frac{1-\tau}{4}\|y-\bar{y}\| \leq \frac{1-\tau}{2}\left\|y_{1}-\bar{y}\right\|
$$

and consequently,

$$
\begin{aligned}
\left\langle y^{*}, y_{1}-\bar{y}\right\rangle & \geq\left\langle y^{*}, y_{2}-\bar{y}\right\rangle-\left\|y_{2}-y_{1}\right\|=\left\|y_{2}-\bar{y}\right\|-\left\|y_{2}-y_{1}\right\| \\
& \geq\left\|y_{1}-\bar{y}\right\|-2\left\|y_{2}-y_{1}\right\|>\tau\left\|y_{1}-\bar{y}\right\| .
\end{aligned}
$$

Making use of (4.9), we obtain

$$
d_{\gamma}\left(\left(0,-y^{*}\right), N_{\mathrm{gph} F_{p}}^{F}\left(x_{1}, y_{1}\right)\right) \leq\left\|\left(0, y^{*}\right)+\left(u^{*}, v^{*}\right)\right\|_{\gamma}<\left\|\left(\hat{x}^{*}, \hat{y}^{*}\right)\right\|_{\gamma}+\varepsilon<\alpha .
$$

This contradicts (4.6).

Remark 4.5. (i) Condition (4.6) with the Fréchet normal cones is obviously weaker (hence, more efficient) than its version with the Clarke ones; cf. Remark 4.2. However, the Asplund space sufficient condition for uniform $\alpha$-subregularity in part (ii) of Theorem 4.4 is not necessarily weaker than its general Banach space version in part (i), as it replaces the equality in (4.7) with a less restrictive inequality in (4.8), which involves an additional parameter $\tau$. Of course, $\tau$ can be chosen arbitrarily close to 1 making the difference between the constraints (4.7) and (4.8) less significant. The weaker than (4.7) conditions (4.8) employed in part (ii) of Theorem 4.4 are due to the approximate subdifferential sum rule (Lemma 2.5(iii)) used in its proof.

(ii) The following alternative sufficient condition has been established half way within the proof of part (ii) of Theorem $4 \cdot 4$ :

$X$ and $Y$ are Asplund, and, given any $\varepsilon>0$, inequality (4.6) holds with $N:=N^{F}$ for all $v \in B_{\varepsilon}(y)$ and all $y^{*} \in Y^{*}$ satisfying (4.7) with $v$ in place of $y$.

It employs the stronger equality conditions (4.7) instead of (4.8), but involves an unknown vector $v$ (arbitrarily close to $y$ ). Conditions of this type are used by some authors, but we prefer more explicit ones in Theorem 4.4(ii) and the statements derived from it.

The qualitative sufficient conditions for uniform regularity follow immediately.

Corollary 4.6. The mapping $F$ is subregular in $x$ uniformly in $p$ over $P$ at $(\bar{x}, \bar{y})$ if one of the following conditions is satisfied:

(i) $\sup _{\gamma>0} \liminf _{\substack{F_{p}^{-1}(\bar{y}) \nexists x \rightarrow \bar{x}, F(p, x) \ni y \rightarrow \bar{y} \\ p \in P, y \neq \bar{y},\left\|y^{*}\right\|=1,\left\langle y^{*}, y-\bar{y}\right\rangle=\|y-\bar{y}\|}} d_{\gamma}\left(\left(0,-y^{*}\right), N_{g p h F_{p}}^{C}(x, y)\right)>0$; 
(ii) $X$ and $Y$ are Asplund, and

$$
\sup _{\gamma>0, \tau \in] 0,1[} \liminf _{\substack{F_{p}^{-1}(\bar{y}) \nexists x \rightarrow \bar{x}, F(p, x) \ni y \rightarrow \bar{y} \\ p \in P, y \neq \bar{y},\left\|y^{*}\right\|=1,\left\langle y^{*}, y-\bar{y}\right\rangle>\tau\|y-\bar{y}\|}} d_{y}\left(\left(0,-y^{*}\right), N_{g p h F_{p}}^{F}(x, y)\right)>0 .
$$

The next example illustrates the sufficient conditions for subregularity in Corollary 4.6.

Example 4.7. Let $P=X=Y:=\mathbb{R}, F(p, x):=\left\{(p-x)^{2}\right\}$ for all $p \in P$ and $x \in X$, and let $\bar{y}:=0$. By (1.3), $F_{p}^{-1}(\bar{y})=\{p\}$. Thus, $d\left(x, F_{p}^{-1}(\bar{y})\right)=|x-p|$ and $d(\bar{y}, F(p, x))=(x-p)^{2}$ for all $p \in P$ and $x \in X$. Hence, for any $\alpha>0$ and $p \in P$, inequality (1.5) is violated when $x$ sufficiently close to $\bar{x}:=0$, i.e. the mapping $F$ is not subregular in $x$ uniformly in $p$ over $P$ at $(\bar{x}, \bar{y})$. Observe that gph $F_{p}$ is closed for all $p \in P$, and, for any $p \in P$ and $(x, y) \in \operatorname{gph} F_{p}$,

$$
(2(x-p),-1) \in N_{\mathrm{gph} F_{p}}^{C}(x, y)=N_{\mathrm{gph} F_{p}}^{F}(x, y) .
$$

Let $p=0, x \neq 0, y=x^{2}$, and $y^{*} \in \mathbb{R}$ satisfy (4.7) or (4.8), hence, $y^{*}=1$, and, for any $\gamma>0$, $d_{\gamma}\left(\left(0,-y^{*}\right),(2 x,-1)\right)=2|x| \rightarrow 0$ as $x \downarrow 0$. Both inequalities in Corollary 4.6 are not satisfied.

Theorem 4.4 yields sufficient conditions for uniform $\alpha$-subregularity in terms of coderivatives.

Corollary 4.8. The mapping $F$ is $\alpha$-subregular in $x$ uniformly in $p$ over $P$ at $(\bar{x}, \bar{y})$ with $\delta$ and $\mu$ if, for some $\eta \in] 0,+\infty]$, and all $p \in P, x \in B_{\delta+\mu}(\bar{x})$ and $y \in Y$ satisfying (3.4), one of the following conditions is satisfied:

(i) with $D^{*}:=D_{C}^{*}$, for all $y^{*} \in Y^{*}$ satisfying $(4 \cdot 7)$, it holds

$$
d\left(0, D^{*} F_{p}(x, y)\left(B_{\eta}\left(y^{*}\right)\right)\right) \geq \alpha
$$

(ii) $X$ and $Y$ are Asplund, and there exists $a \tau \in] 0,1\left[\right.$ such that inequality (4.10) holds with $D^{*}:=D_{F}^{*}$ for all $y^{*} \in Y^{*}$ satisfying (4.8).

Proof. Given an $\eta \in] 0,+\infty]$, set $\gamma:=\alpha^{-1} \eta$. In view of the representations (2.5) of the coderivative and (4.2) of the dual norm, condition (4.6) means that $\left\|u^{*}\right\|+\gamma^{-1}\left\|v^{*}-y^{*}\right\| \geq \alpha$ for all $v^{*} \in Y^{*}$ and $u^{*} \in D^{*} F_{p}(x, y)\left(v^{*}\right)$. The last inequality is obviously satisfied if either $\left\|u^{*}\right\| \geq \alpha$ or $\left\|v^{*}-y^{*}\right\| \geq \eta$, or equivalently, if $\left\|u^{*}\right\| \geq \alpha$ when $v^{*} \in B_{\eta}\left(y^{*}\right)$.

The coderivative sufficient condition (4.10) can be replaced by its 'normalized' (and a little stronger!) version.

Corollary 4.9. The mapping $F$ is $\alpha$-subregular in $x$ uniformly in $p$ over $P$ at $(\bar{x}, \bar{y})$ with $\delta$ and $\mu$ if, for some $\eta \in] 0,1\left[\right.$, and all $p \in P, x \in B_{\delta+\mu}(\bar{x})$ and $y \in Y$ satisfying (3.4), one of the following conditions is satisfied:

(i) with $D^{*}:=D_{C}^{*}$,

$$
d\left(0, D^{*} F_{p}(x, y)\left(\frac{v^{*}}{\left\|v^{*}\right\|}\right)\right) \geq \frac{\alpha}{1-\eta}
$$

for all $y^{*} \in Y^{*}$ satisfying (4.7) and $v^{*} \in B_{\eta}\left(y^{*}\right)$;

(ii) $X$ and $Y$ are Asplund, and there exists a $\tau \in] 0,1\left[\right.$ such that inequality (4.11) holds with $D^{*}:=D_{F}^{*}$ for all $y^{*} \in Y^{*}$ satisfying (4.8) and $v^{*} \in B_{\eta}\left(y^{*}\right)$.

Proof. Let $\eta \in] 0,1\left[, p \in P, x \in B_{\delta+\mu}(\bar{x})\right.$ and $y \in Y$ satisfy (3.4), and $y^{*} \in Y^{*}$ satisfy either (4.7) or (4.8). We need to show that, if inequality (4.11) holds for all $v^{*} \in B_{\eta}\left(y^{*}\right)$, then inequality (4.10) holds. First note that, in view of (4.7) or (4.8), $\left\|y^{*}\right\|=1$. Let $v^{*} \in B_{\eta}\left(y^{*}\right)$ and $u^{*} \in D^{*} F_{p}(x, y)\left(v^{*}\right)$. Then $\left.\left.\left\|v^{*}\right\|>1-\eta \in\right] 0,+\infty\right]$. Thus, condition (4.11) is well defined. Moreover, $u^{*} /\left\|v^{*}\right\| \in D^{*} F_{p}(x, y)\left(v^{*} /\left\|v^{*}\right\|\right)$ and, in view of (4.11), $\left\|u^{*}\right\| \geq \alpha\left\|v^{*}\right\| /(1-\eta)>\alpha$, i.e. inequality (4.10) holds. 
The next qualitative assertion is an immediate consequence of Corollary 4.8.

Corollary 4.10. The mapping $F$ is subregular in $x$ uniformly in $p$ over $P$ at $(\bar{x}, \bar{y})$ if one of the following conditions is satisfied:

(i) $\lim _{\delta \downarrow 0} \inf _{x \in B_{\delta}(\bar{x}) \backslash F_{p}^{-1}(\bar{y}), \bar{y} \neq y \in F(p, x) \cap B_{\delta}(\bar{y})} d\left(0, D_{C}^{*} F_{p}(x, y)\left(B_{\delta}\left(y^{*}\right)\right)\right)>0$; $p \in P,\left\|y^{*}\right\|=1,\left\langle y^{*}, y-\bar{y}\right\rangle=\|y-\bar{y}\|$

(ii) $X$ and $Y$ are Asplund, and

$$
\lim _{\substack { \delta \downarrow, \tau \uparrow 1 \\
\begin{subarray}{c}{x \in B_{\delta}(\bar{x}) \backslash F_{p}^{-1}(\bar{y}), \bar{y} \neq y \in F(p, x) \cap B_{\delta}(\bar{y}) \\
p \in P,\left\|y^{*}\right\|=1,\left\langle y^{*}, y-\bar{y}\right\rangle>\tau\|y-\bar{y}\|{ \delta \downarrow , \tau \uparrow 1 \\
\begin{subarray} { c } { x \in B _ { \delta } ( \overline { x } ) \backslash F _ { p } ^ { - 1 } ( \overline { y } ) , \overline { y } \neq y \in F ( p , x ) \cap B _ { \delta } ( \overline { y } ) \\
p \in P , \| y ^ { * } \| = 1 , \langle y ^ { * } , y - \overline { y } \rangle > \tau \| y - \overline { y } \| } }\end{subarray}} d\left(0, D_{F}^{*} F_{p}(x, y)\left(B_{\delta}\left(y^{*}\right)\right)\right)>0 .
$$

Remark 4.11. (i) In the case when $P$ is a neighborhood of a given point $\bar{p}$ in a metric space, Corollary 4.10 (taking into account Remark 4.5(ii) in some instances) improves [40, Theorem 3.6], [47, Theorem 3.4], [41, Theorem 3.2], [29, Theorem 3.5], [28, Theorem 3.1], [50, Corollary 2.2], [14, Theorem 1] (in the linear setting), and [32, Theorem 4.1(e)].

(ii) Clarke normal cones in this section can be replaced by Ioffe's G-normal cones [33].

\subsection{DUAL NECESSARY CONDITIONS}

In this subsection, $X$ and $Y$ are normed spaces, $F: P \times X \rightrightarrows Y, \bar{x} \in X, \bar{y} \in Y, \alpha>0, \delta \in] 0,+\infty]$, $\mu \in] 0,+\infty]$, and we assume that $g p h F_{p}$ is convex for all $p \in P$.

The next statement provides a necessary condition for uniform $\alpha$-subregularity in terms of subdifferentials of the function $\psi_{p}$ defined by (3.1).

Proposition 4.12. If $F$ is $\alpha$-subregular in $x$ uniformly in $p$ over $P$ at $(\bar{x}, \bar{y})$ with $\delta$ and $\mu$, then inequality (4.3) is satisfied with $\gamma:=\alpha^{-1}$ for all $p \in P, x \in B_{\delta}(\bar{x})$ and $y \in Y$ satisfying (3.4).

Proof. Under the assumptions made, the function $\psi_{p}$ is convex for all $p \in P$. Let $\gamma:=\alpha^{-1}$, and $p \in P$, $x \in B_{\delta}(\bar{x})$ and $y \in Y$ satisfy (3.4). For any $\left(x^{*}, y^{*}\right) \in \partial \psi_{p}(x, y)$, we have

$$
\begin{aligned}
\left\|\left(x^{*}, y^{*}\right)\right\|_{\gamma}= & \sup _{\substack{(u, v) \neq(0,0)\\
}} \frac{\left\langle\left(x^{*}, y^{*}\right),(u, v)\right\rangle}{\|(u, v)\|_{\gamma}} \\
= & \lim _{\substack{u \rightarrow x, v \rightarrow y \\
(u, v) \neq(x, y)}} \frac{-\left\langle\left(x^{*}, y^{*}\right),(u, v)-(x, y)\right\rangle}{\|(u, v)-(x, y)\|_{\gamma}} \\
\geq & \limsup _{\substack{u \rightarrow x, v \rightarrow y \\
(u, v) \neq(x, y)}} \frac{\psi_{p}(x, y)-\psi_{p}(u, v)}{\|(u, v)-(x, y)\|_{\gamma}} \\
= & \limsup _{\substack{u \rightarrow x, v \rightarrow y \\
(u, v) \in \operatorname{gph} F_{p},(u, v) \neq(x, y)}} \frac{\|y-\bar{y}\|-\|v-\bar{y}\|}{\|(u, v)-(x, y)\|_{\gamma}} .
\end{aligned}
$$

By Corollary 3.4(i), we have $\left\|\left(x^{*}, y^{*}\right)\right\|_{\gamma} \geq \alpha$. Taking the infimum in the left-hand side of the last inequality over $\left(x^{*}, y^{*}\right) \in \partial \psi_{p}(x, y)$, we obtain inequality (4.3).

Combining the above statement with Proposition 4.1, we obtain a complete subdifferential characterization of the uniform subregularity in the convex setting.

Corollary 4.13. Let $X$ and $Y$ be Banach, and $g p h F_{p}$ be closed for all $p \in P$. The mapping $F$ is subregular in $x$ uniformly in $p$ over $P$ at $(\bar{x}, \bar{y})$ if and only if

$$
\sup _{\gamma>0} \liminf _{\substack{x \rightarrow \bar{x}, y \rightarrow \bar{y} \\ p \in P, x \notin F_{p}^{-1}(\bar{y}), \bar{y} \neq y \in F(p, x)}} d_{\gamma}\left(0, \partial \psi_{p}(x, y)\right)>0 .
$$


The next corollary follows from Proposition 4.12 in view of the representation (4.2) of the dual norm. Corollary 4.14. If $F$ is $\alpha$-subregular in $x$ uniformly in $p$ over $P$ at $(\bar{x}, \bar{y})$ with $\delta$ and $\mu$, then $\left\|x^{*}\right\| \geq$ $\alpha\left(1-\left\|y^{*}\right\|\right)$ for all $p \in P, x \in B_{\delta}(\bar{x})$ and $y \in Y$ satisfying $(3.4)$, and all $\left(x^{*}, y^{*}\right) \in \partial \psi_{p}(x, y)$. As a consequence,

$$
\liminf _{\substack{F_{p}^{-1}(\bar{y}) \nexists x \rightarrow \bar{x}, F(p, x) \\ p \in P, y \neq y \rightarrow\left(x^{*}, y^{*}\right) \in \partial \psi_{p}(x, y)}}\left\|x^{*}\right\| \geq \alpha .
$$

The next statement gives a partial converse to Theorem 4.4 .

Theorem 4.15. If $F$ is $\alpha$-subregular in $x$ uniformly in $p$ over $P$ at $(\bar{x}, \bar{y})$ with $\delta$ and $\mu$, then for all $p \in P$, $x \in B_{\delta}(\bar{x})$ and $y \in Y$ satisfying (3.4), and all $y^{*} \in Y^{*}$ satisfying (4.7), inequality (4.6) is satisfied with $\gamma:=\alpha^{-1}$.

Proof. Observe that $\psi_{p}$ is the sum of the convex continuous function $v \mapsto g(v):=\|v-\bar{y}\|$ and the indicator function of the convex set gph $F_{p}$. By Lemma 2.5(i), $\partial \psi_{p}(x, y)=\{0\} \times \partial g(y)+N_{g p h} F_{p}(x, y)$. The assertion follows from Proposition 4.12 in view of Lemma 2.2(ii).

Combining Theorems 4.4 and 4.15 , we can formulate a necessary and sufficient characterization of the uniform subregularity in the convex setting.

Corollary 4.16. Let $X$ and $Y$ be Banach, and $g p h F_{p}$ be closed for all $p \in P$. The mapping $F$ is subregular in $x$ uniformly in $p$ over $P$ at $(\bar{x}, \bar{y})$ if and only if

$$
\sup _{\gamma>0} \liminf _{\substack{F_{p}^{-1}(\bar{y}) \nexists x \rightarrow \bar{x}, F(p, x) \ni y \rightarrow \bar{y} \\ p \in P, y \neq \bar{y},\left\|y^{*}\right\|=1,\left\langle y^{*}, y-\bar{y}\right\rangle=\|y-\bar{y}\|}} d_{\gamma}\left(\left(0,-y^{*}\right), N_{g p h F_{p}}(x, y)\right)>0 .
$$

The next example illustrates the necessary condition in Theorem 4.15.

Example 4.17. Let $P=X=Y:=\mathbb{R}, F(p, x):=\{p-x\}$ for all $p \in P$ and $x \in X$, and let $\bar{x}=\bar{y}:=0$. By (1.3), $F_{p}^{-1}(\bar{y})=\{p\}$. Thus, $d\left(x, F_{p}^{-1}(\bar{y})\right)=d(\bar{y}, F(p, x))=|x-p|$ for all $p \in P$ and $x \in X$. Hence, inequality (1.5) is satisfied for all $p \in P, x \in X$, and $\alpha \in] 0,1]$, i.e. the mapping $F$ is $\alpha$-subregular in $x$ uniformly in $p$ over $P$ at $(\bar{x}, \bar{y})$ for any $\alpha \in] 0,1]$. We have gph $F_{p}=\{(x, y) \mid y=p-x\}$ is closed and convex for all $p \in P$, and $N_{\operatorname{gph} F_{p}}(x, y)=\{(t, t) \mid t \in \mathbb{R}\}$ for any $(x, y) \in \operatorname{gph} F_{p}$. Let $y^{*} \in \mathbb{R}$ satisfy (4.7). Then $y^{*}=1$ if $y>0$, and $y^{*}=-1$ if $y<0$. It is easy to check that, given a $\gamma>0$, in both cases the distance $d_{\gamma}\left(\left(0,-y^{*}\right), N_{\mathrm{gph} F_{p}}(x, y)\right)$ equals 1 if $\gamma \leq 1$, or $\gamma^{-1}$ if $\gamma>1$. Hence, condition (4.12) is satisfied, confirming the uniform subregularity of $F$.

The next statement is a consequence of Theorem 4.15. It is in a sense a partial converse to Corollary 4.8. Corollary 4.18. If $F$ is $\alpha$-subregular in $x$ uniformly in $p$ over $P$ at $(\bar{x}, \bar{y})$ with $\delta$ and $\mu$, then $d\left(0, D^{*} F_{p}(x, y)\left(B_{\eta}\left(y^{*}\right)\right)\right) \geq \alpha(1-\eta)$ for all $\left.\eta \in\right] 0,1\left[, p \in P, x \in B_{\delta}(\bar{x})\right.$ and $y \in Y$ satisfying (3.4), and all $y^{*} \in Y^{*}$ satisfying (4.7).

Proof. In view of the representations (2.5) of the coderivative and (4.2) of the dual norm, condition (4.6) with $\gamma:=\alpha^{-1}$ means that $\left\|u^{*}\right\|+\alpha\left\|v^{*}-y^{*}\right\| \geq \alpha$ for all $v^{*} \in Y^{*}$ and $u^{*} \in D^{*} F_{p}(x, y)\left(v^{*}\right)$. Hence, it yields $\left\|u^{*}\right\|>\alpha(1-\eta)$ if $\left\|v^{*}-y^{*}\right\|<\eta$.

Combining the above statement with Corollary 4.8 , we obtain a complete coderivative characterization of the uniform $\alpha$-subregularity in the convex setting. It improves [14, Theorem 3] (in the linear case).

Corollary 4.19. Let $X$ and $Y$ be Banach, and $g p h F_{p}$ be closed for all $p \in P$. The mapping $F$ is $\alpha$-subregular in $x$ uniformly in $p$ over $P$ at $(\bar{x}, \bar{y})$ if and only if

$$
\lim _{\delta \downarrow 0} \inf _{\substack{x \in B_{\delta}(\bar{x}) \backslash F_{p}^{-1}(\bar{y}), \bar{y} \neq y \in F(p, x) \cap B_{\delta}(\bar{y}) \\ p \in P,\left\|y^{*}\right\|=1,\left\langle y^{*}, y-\bar{y}\right\rangle=\|y-\bar{y}\|}} d\left(0, D^{*} F_{p}(x, y)\left(B_{\delta}\left(y^{*}\right)\right)\right) \geq \alpha .
$$




\section{METRIC SUBREGULARITY, METRIC REGULARITY AND IMPLICIT MULTIFUNCTIONS}

In this section, we illustrate the necessary and sufficient conditions for the uniform subregularity established in the preceding sections by characterizing several conventional properties of set-valued mappings.

\subsection{METRIC SUBREGULARITY}

As observed in the Introduction, the conventional regularity properties in Definition 1.1 are particular cases of the uniform regularity properties in Definition 1.2 corresponding to $P$ being a singleton, which practically means that the set-valued mapping $F$ does not involve a parameter.

The next three statements, which are immediate consequences of the corresponding 'parametric' ones in Sections 3 and 4 , illustrate this observation for the case of subregularity. Here $X$ and $Y$ are normed spaces, $F: X \rightrightarrows Y,(\bar{x}, \bar{y}) \in \operatorname{gph} F, \alpha>0, \delta \in] 0,+\infty]$ and $\mu \in] 0,+\infty]$.

Proposition 5.1. (i) Suppose gph $F$ is convex. If $F$ is $\alpha-$ subregular at $(\bar{x}, \bar{y})$ with $\delta$ and $\mu$, then

$$
\limsup _{\substack{u \rightarrow x, v \rightarrow y,(u, v) \in g p h F,(u, v) \neq(x, y) \\ d(u, \bar{x}<\delta+\mu, d(v, \bar{y})<\alpha \mu}} \frac{\|y-\bar{y}\|-\|v-\bar{y}\|}{\|(u-x, v-y)\|_{\gamma}} \geq \alpha
$$

for $\gamma:=\alpha^{-1}$, and all $x \in B_{\delta}(\bar{x})$ and $y \in Y$ satisfying

$$
x \notin F^{-1}(\bar{y}), \quad y \in F(x) \cap B_{\alpha \mu}(\bar{y}) .
$$

(ii) Suppose $X$ and $Y$ are Banach, and gph $F$ is closed. If inequality (5.1) holds for some $\gamma>0$, and all $x \in B_{\delta+\mu}(\bar{x})$ and $y \in Y$ satisfying (5.2), then $F$ is $\alpha$-subregular at $(\bar{x}, \bar{y})$ with $\delta$ and $\mu$.

Proof. The statement is a consequence of Corollary 3.4.

Proposition 5.2. $\quad$ (i) Suppose gph $F$ is convex. If $F$ is $\alpha$-subregular at $(\bar{x}, \bar{y})$ with $\delta$ and $\mu$, then

$$
d_{y}\left(\left(0,-y^{*}\right), N_{g p h F}(x, y)\right) \geq \alpha
$$

for $y:=\alpha^{-1}$, all $x \in B_{\delta}(\bar{x})$ and $y \in Y$ satisfying (5.2), and all $y^{*} \in Y^{*}$ satisfying (4.7).

(ii) Suppose $X$ and $Y$ are Banach, and gph $F$ is closed. The mapping $F$ is $\alpha$-subregular at $(\bar{x}, \bar{y})$ with $\delta$ and $\mu$ if, for some $\gamma>0$, and all $x \in B_{\delta+\mu}(\bar{x})$ and $y \in Y$ satisfying (5.2), one of the following conditions is satisfied:

(a) inequality (5.3) holds with $N:=N^{C}$ for all $y^{*} \in Y^{*}$ satisfying (4.7);

(b) $X$ and $Y$ are Asplund, and there exists a $\tau \in] 0,1\left[\right.$ such that inequality (5.3) holds with $N:=N^{F}$ for all $y^{*} \in Y^{*}$ satisfying (4.8).

Proof. The statement is a consequence of Theorems 4.4 and 4.15 .

Proposition 5.3. (i) Suppose gph $F$ is convex. If $F$ is $\alpha$-subregular at $(\bar{x}, \bar{y})$ with $\delta$ and $\mu$, then $d\left(0, D^{*} F(x, y)\left(B_{\eta}\left(y^{*}\right)\right)\right) \geq \alpha(1-\eta)$ for any $\left.\eta \in\right] 0,1\left[\right.$, all $x \in B_{\delta}(\bar{x})$ and $y \in Y$ satisfying (5.2), and all $y^{*} \in Y^{*}$ satisfying (4.7).

(ii) Suppose $X$ and $Y$ are Banach, and $g p h F$ is closed. The mapping $F$ is $\alpha$-subregular at $(\bar{x}, \bar{y})$ with $\delta$ and $\mu$ if, for some $\eta \in] 0,+\infty]$, and all $x \in B_{\delta+\mu}(\bar{x})$ and $y \in Y$ satisfying (5.2), one of the following conditions is satisfied: 
(a) with $D^{*}:=D_{C}^{*}$, for all $y^{*} \in Y^{*}$ satisfying (4.7), it holds

$$
d\left(0, D^{*} F(x, y)\left(B_{\eta}\left(y^{*}\right)\right)\right) \geq \alpha ;
$$

(b) $X$ and $Y$ are Asplund, and there exists a $\tau \in] 0,1\left[\right.$ such that inequality (5.4) holds with $D^{*}:=D_{F}^{*}$ for all $y^{*} \in Y^{*}$ satisfying (4.8).

Proof. The statement is a consequence of Corollaries 4.8 and 4.18 .

Remark 5.4. (i) In Proposition 5.1(ii), it is sufficient to assume that $X$ and $Y$ are complete metric spaces (with distances in place of norms in condition (5.1)), or even that gph $F$ is complete; cf. Remark 3.2(iii). In this setting, the sufficient condition in Proposition 5.1(ii) can be viewed as a quantitative version of [38, Corollary 5.8(d)] and [32, Theorem 2.4(a)].

(ii) Proposition 5.3 improves [42, Theorem 5.3]. In the linear setting, part (ii) of this proposition improves [42, Theorem 3.3], [49, Theorem 6], [14, Theorem 8], [32, Theorem 2.6], and the corresponding parts of [38, Corollary 5.8]. Proposition 5.3(ii) with condition (a) recaptures [63, Theorem 3.2].

\subsection{METRIC REGULARITY}

The conventional metric regularity is a particular case of the uniform regularity property in Definition 1.2(i) corresponding to $P$ being a singleton. At the same time, as it follows from the observation in Remark 1.3, in the normed space setting it can be treated as a particular case of the uniform subregularity property in Definition 1.2(ii) for the set-valued mapping $\widehat{F}(y, x):=F(x)-y,(y, x) \in Y \times X$ with $y$ considered as a parameter. Obviously $(\bar{x}, \bar{y}) \in \operatorname{gph} F$ if and only if $(\bar{y}, \bar{x}, 0) \in \operatorname{gph} \widehat{F}$.

The next three statements, which are immediate consequences of the corresponding 'parametric' ones in Sections 3 and 4 , illustrate the above observation. Here $X$ and $Y$ are normed spaces, $F: X \rightrightarrows Y$, $(\bar{x}, \bar{y}) \in \operatorname{gph} F, \alpha>0, \delta \in] 0,+\infty]$ and $\mu \in] 0,+\infty]$.

Proposition 5.5. $\quad$ (i) Suppose gph $F$ is convex. If $F$ is $\alpha$-regular at $(\bar{x}, \bar{y})$ with $\delta$ and $\mu$, then

$$
\limsup _{\substack{u \rightarrow x, v \rightarrow z,(u, v) \in g p h F,(u, v) \neq(x, z) \\ d(u, \bar{x})<\delta+\mu, d(v, y)<\alpha \mu}} \frac{\|z-y\|-\|v-y\|}{\|(u-x, v-z)\|_{\gamma}} \geq \alpha
$$

for $\gamma:=\alpha^{-1}$, and all $x \in B_{\delta}(\bar{x}), y \in B_{\delta}(\bar{y})$ and $z \in Y$ satisfying

$$
x \notin F^{-1}(y), \quad z \in F(x) \cap B_{\alpha \mu}(y) .
$$

(ii) Suppose $X$ and $Y$ are Banach spaces, and gph $F$ is closed. The mapping $F$ is $\alpha$-regular at $(\bar{x}, \bar{y})$ with $\delta$ and $\mu$ if inequality (5.5) holds with some $\gamma>0$ for all $x \in B_{\delta+\mu}(\bar{x}), y \in B_{\delta}(\bar{y})$ and $z \in Y$ satisfying (5.6).

Proof. The statement is a consequence of Corollary 3.4.

Proposition 5.6. (i) Suppose gph $F$ is convex. If $F$ is $\alpha$-regular at $(\bar{x}, \bar{y})$ with $\delta$ and $\mu$, then

$$
d_{\gamma}\left(\left(0,-y^{*}\right), N_{g p h F}(x, z)\right) \geq \alpha
$$

for $y:=\alpha^{-1}$, and all $x \in B_{\delta}(\bar{x}), y \in B_{\delta}(\bar{y})$ and $z \in Y$ satisfying (5.6), and $y^{*} \in Y^{*}$ satisfying

$$
\left\|y^{*}\right\|=1, \quad\left\langle y^{*}, z-y\right\rangle=\|z-y\| .
$$


(ii) Suppose $X$ and $Y$ are Banach, and $g p h F$ is closed. The mapping $F$ is $\alpha$-regular at $(\bar{x}, \bar{y})$ with $\delta$ and $\mu$ if, for some $\gamma>0$, and all $x \in B_{\delta+\mu}(\bar{x}), y \in B_{\delta}(\bar{y})$ and $z \in Y$ satisfying (5.6), one of the following conditions holds:

(a) inequality (5.7) holds with $N:=N^{C}$ for all $y^{*} \in Y^{*}$ satisfying (5.8);

(b) $X$ and $Y$ are Asplund, and there exists $a \tau \in] 0,1\left[\right.$ such that inequality (5.7) holds with $N:=N^{F}$ for all $y^{*} \in Y^{*}$ satisfying

$$
\left\|y^{*}\right\|=1, \quad\left\langle y^{*}, z-y\right\rangle>\tau\|z-y\| .
$$

Proof. The statement is a consequence of Theorems 4.4 and 4.15 .

Proposition 5.7. (i) Suppose gph $F$ is convex. If $F$ is $\alpha$-regular at $(\bar{x}, \bar{y})$ with $\delta$ and $\mu$, then $d\left(0, D^{*} F(x, z)\left(B_{\eta}\left(y^{*}\right)\right)\right) \geq \alpha(1-\eta)$ for all $\left.\eta \in\right] 0,1\left[, x \in B_{\delta}(\bar{x}), y \in B_{\delta}(\bar{y})\right.$ and $z \in Y$ satisfying (5.6), and all $y^{*} \in Y^{*}$ satisfying (5.8).

(ii) Suppose $X$ and $Y$ are Banach, and gph $F$ is closed. The mapping $F$ is $\alpha$-regular at $(\bar{x}, \bar{y})$ with $\delta$ and $\mu$ if, for some $\eta \in] 0,+\infty]$ and all $\left.x \in B_{\delta+\mu} \bar{x}\right), y \in B_{\delta}(\bar{y})$ and $z \in Y$ satisfying (5.6), one of the following conditions holds:

(a) with $D^{*}:=D_{C}^{*}$, for all $y^{*} \in Y^{*}$ satisfying (5.8), it holds

$$
d\left(0, D^{*} F(x, z)\left(B_{\eta}\left(y^{*}\right)\right)\right) \geq \alpha ;
$$

(b) $X$ and $Y$ are Asplund, and there exists a $\tau \in] 0,1[$ such that inequality (5.10) holds with $D^{*}:=D_{F}^{*}$ for all $y^{*} \in Y^{*}$ satisfying (5.9).

Proof. The statement is a consequence of Corollaries 4.8 and 4.18 .

Remark 5.8. (i) In the normed space setting, the sufficient condition in Proposition 5.5(ii) can be viewed as a quantitative version of [30, Theorem 1] and [32, Theorem 2.4(a)].

(ii) Proposition 5.7(ii) enhances [13, Theorem 3.7]. Proposition 5.7(ii) with condition (a) improves [28, Corollary 3.1] and [13, Theorem 3.5] (in the linear case), while with condition (b) it improves (in the linear case) [13, Theorem 3.1] and [14, Theorem 7].

\section{$5 \cdot 3$ IMPLICIT MULTIFUNCTIONS}

Now we get back to the implicit multifunction (1.3) and consider its particular case corresponding to the parametric inclusion $\bar{y} \in F(p, x)$ (with fixed left-hand side), i.e.

$$
G(p):=\{x \in X \mid \bar{y} \in F(p, x)\}, \quad p \in P,
$$

where $F: P \times X \rightrightarrows Y$, and $P, X$ and $Y$ are metric spaces. Stability properties of implicit multifunctions, i.e. solution sets of parametric inclusions, are of great importance for many applications and have been the subject of numerous publications; cf., e.g., [6-8,11,13-15, 22, 25, 28, 29, 32, 33,35,40,41,47,49,50,52,54, 58, 6o]. Here, for illustration, we focus on the most well known perturbation stability property of set-valued mappings called Aubin property; cf. [22, 45].

Definition 5.9. A mapping $G: P \rightrightarrows X$ between metric spaces has the Aubin property at $(\bar{p}, \bar{x}) \in \operatorname{gph} G$ with rate $l>0$ if there exist $\eta \in] 0,+\infty], \delta \in] 0,+\infty]$ and $\mu \in] 0,+\infty]$ such that

$$
d(x, G(p)) \leq l d\left(p, p^{\prime}\right)
$$

for all $p, p^{\prime} \in B_{\eta}(\bar{p})$ with $d\left(p, p^{\prime}\right)<\mu$, and $x \in G\left(p^{\prime}\right) \cap B_{\delta}(\bar{x})$. 
Similar to Definitions 1.1, 1.2 and 1.4, the inequality $d\left(p, p^{\prime}\right)<\mu$ is not essential in the above definition and can be dropped together with the constant $\mu$. We keep them for consistency with the definitions and characterizations in the preceding sections. We also establish connections between the constant $\mu$ and the corresponding constants in the other definitions.

Given a point $(\bar{p}, \bar{x}, \bar{y}) \in \operatorname{gph} F$ and a number $\alpha>0$, the uniform $\alpha$-subregularity property of $F$ at $(\bar{p}, \bar{x}, \bar{y})$ in Definition 1.4(ii) means that there exist $\eta \in] 0,+\infty], \delta \in] 0,+\infty]$ and $\mu \in] 0,+\infty]$ such that

$$
\alpha d(x, G(p)) \leq d(\bar{y}, F(p, x))
$$

for all $p \in B_{\eta}(\bar{p})$ and $x \in B_{\delta}(\bar{x})$ with $d(\bar{y}, F(p, x))<\alpha \mu$. Several primal and dual sufficient and necessary conditions for this property have been formulated in the preceding sections.

Inequality (5.12) provides an estimate for the distance from $x$ to the value of the implicit multifunction (5.11) at $p$ in terms of the residual of the parametric inclusion. However, this estimate does not say much about the behaviour of the implicit multifunction. An additional assumption on the mapping $F$ is needed, which would allow one to get rid of $F$ in the right-hand side of the inequality (5.12). This additional assumption is given in the next definition, which is a modification of the second part of [32, Definition 3.1], where we borrow the terminology from. A similar property was considered in [35], where the authors used the name Lipschitz lower semicontinuity.

Definition 5.10. Let $l>0$. The mapping $F$ is said to $l$-recede in $p$ uniformly in $x$ at $(\bar{p}, \bar{x}, \bar{y})$ if there exist $\eta \in] 0,+\infty], \delta \in] 0,+\infty]$ and $\mu \in] 0,+\infty]$ such that

$$
d(\bar{y}, F(p, x)) \leq l d\left(p, p^{\prime}\right)
$$

for all $x \in B_{\delta}(\bar{x})$ and $p, p^{\prime} \in B_{\eta}(\bar{p})$ with $d\left(p, p^{\prime}\right)<\mu$ and $\bar{y} \in F\left(p^{\prime}, x\right)$.

In what follows we assume that $(\bar{p}, \bar{x}, \bar{y}) \in \operatorname{gph} F, \alpha>0, l>0, \eta \in] 0,+\infty], \delta \in] 0,+\infty]$ and $\mu \in] 0,+\infty]$. The next statement is a modification of [32, Theorem 3.2].

Proposition 5.11. Suppose that $F$

- is $\alpha$-subregular in $x$ uniformly in $p$ at $(\bar{p}, \bar{x}, \bar{y})$ with $\eta, \delta$ and $\mu$;

- $l$-recedes in $p$ uniformly in $x$ at $(\bar{p}, \bar{x}, \bar{y})$ with $\eta, \delta$ and $\mu^{\prime}:=\alpha \mu / l$.

Then the mapping $G$ given by (5.11) has the Aubin property at $(\bar{p}, \bar{x})$ with rate $l / \alpha$, and $\eta, \delta$ and $\mu^{\prime}$.

Proof. Let $p, p^{\prime} \in B_{\eta}(\bar{p})$ with $d\left(p, p^{\prime}\right)<\mu^{\prime}$, and $x \in G\left(p^{\prime}\right) \cap B_{\delta}(\bar{x})$. By (5.11) and (5.13), $\bar{y} \in F\left(p^{\prime}, x\right)$ and $d(\bar{y}, F(p, x))<\alpha \mu$. Using successively (5.12) and (5.13), we obtain

$$
d(x, G(p)) \leq \frac{1}{\alpha} d(\bar{y}, F(p, x)) \leq \frac{l}{\alpha} d\left(p, p^{\prime}\right) .
$$

The proof is completed.

Combining Proposition 5.11 with the sufficient conditions for the uniform subregularity formulated in the preceding sections, we can immediately obtain various sufficient conditions for the Aubin property of the implicit multifunction (5.11). The next proposition collects three sufficient conditions arising from Corollary 3.4(ii), Theorem 4.4 and Corollary 4.8 , respectively.

Proposition 5.12. Let $P$ be a metric space, $X$ and $Y$ be complete metric spaces, $F: P \times X \rightrightarrows Y$ and $G: P \rightrightarrows X$ be given by (5.11). Suppose that $g p h F_{p}$ is closed for all $p \in B_{\eta}(\bar{p})$. The mapping $G$ has the Aubin property at $(\bar{p}, \bar{x})$ with rate $l>0$, and $\eta, \delta$ and $\mu$ if, for some $l^{\prime}>0$, the mapping $F l^{\prime}$-recedes in $p$ uniformly in $x$ at $(\bar{p}, \bar{x}, \bar{y})$ with $\eta, \delta$ and $\mu$, and one of the following conditions holds true: 
(i) there exists $a \gamma>0$ such that

$$
\limsup _{\substack{u \rightarrow x, v \rightarrow y,(u, v) \in g p h F_{p},(u, v) \neq(x, y) \\ d(u, \bar{x})<\delta+l \mu, d(v, \bar{y})<l^{\prime} \mu}} \frac{d(y, \bar{y})-d(v, \bar{y})}{d_{y}((u, v),(x, y))} \geq \frac{l^{\prime}}{l}
$$

for all $p, x$ and $y$ satisfying

$$
p \in B_{\eta}(\bar{p}), x \in B_{\delta+\mu}(\bar{x}) \backslash F_{p}^{-1}(\bar{y}), y \in F(p, x) \cap B_{l^{\prime} \mu}(\bar{y})
$$

(ii) $X$ and $Y$ are Banach, and there exists a $\gamma>0$ such that, with $N:=N^{C}$,

$$
d_{\gamma}\left(\left(0,-y^{*}\right), N_{g p h F_{p}}(x, y)\right) \geq \frac{l^{\prime}}{l}
$$

for all $p, x$ and $y$ satisfying (5.14), and all $y^{*} \in Y^{*}$ satisfying (4.7);

(iii) $X$ and $Y$ are Asplund, and there exist $a \gamma>0$ and $a \tau \in] 0,1$ [ such that condition (5.15) is satisfied with $N:=N^{F}$ for all $p, x$ and $y$ satisfying (5.14), and all $y^{*} \in Y^{*}$ satisfying (4.8);

(iv) $X$ and $Y$ are Banach, and

$$
d\left(0, D^{*} F_{p}(x, y)\left(B_{\eta}\left(y^{*}\right)\right)\right) \geq \frac{l^{\prime}}{l}
$$

with $D^{*}:=D_{C}^{*}$ for all $p, x$ and $y$ satisfying (5.14), and all $y^{*} \in Y^{*}$ satisfying (4.7);

(v) $X$ and $Y$ are Asplund, and there exists a $\tau \in] 0,1\left[\right.$ such that condition (5.16) is satisfied with $D^{*}:=D_{F}^{*}$ for all $p, x$ and $y$ satisfying (5.14), and all $y^{*} \in Y^{*}$ satisfying (4.8).

Remark 5.13. Conditions (i) in Proposition 5.12 can be seen as a quantitative version of [32, Theorem 3.9], while conditions (iv) and (v) improve [32, Theorem 4.1] and [33, Theorem 7.26]. Conditions (ii) and (iii) are new. Note that these two conditions are weaker than conditions (iv) and (v), respectively.

\section{REFERENCES}

[1] S. Adly, R. Cibulka, and H. V. Ngai, Newton's method for solving inclusions using set-valued approximations, SIAM F. Optim. 25 (2015), 159-184, doi:10.1137/130926730.

[2] M. Apetrii, M. Durea, and R. Strugariu, On subregularity properties of set-valued mappings, Set-Valued Var. Anal. 21 (2013), 93-126, doi:10.1007/s11228-012-0213-4.

[3] F. J. Aragón Artacho, A. L. Dontchev, M. Gaydu, M. H. Geoffroy, and V. M. Veliov, Metric regularity of Newton's iteration, SIAM f. Control Optim. 49 (2011), 339-362, doi:10.1137/100792585.

[4] F. J. Aragón Artacho, A.L. Dontchev, and M.H. Geoffroy, Convergence of the proximal point method for metrically regular mappings, in CSVAA 2004-Control, Set-Valued Analysis and Applications, volume 17 of ESAIM Proc., EDP Sci., Les Ulis, 2007, 1-8, doi:10.1051/proc:071701.

[5] T. Aspelmeier, C. Charitha, and D. R. Luke, Local linear convergence of the ADMM/DouglasRachford algorithms without strong convexity and application to statistical imaging, SIAM $\mathcal{F}$. Imaging Sci. 9 (2016), 842-868, doi:10.1137/15m103580x.

[6] D. Azé and S. Benahmed, On implicit multifunction theorems, Set-Valued Anal. 16 (2008), 129-155, doi:10.1007/s11228-008-0072-1. 
[7] D. Azé, J. N. Corvellec, and R. E. Lucchetti, Variational pairs and applications to stability in nonsmooth analysis, Nonlinear Anal. 49 (2002), 643-670, doi:10.1016/so362-546x(01)00129-8.

[8] J. M. Borwein, Stability and regular points of inequality systems, f. Optim. Theory Appl. 48 (1986), 9-52.

[9] J. M. Borwein and Q.J. Zhu, Techniques of Variational Analysis, Springer, New York, 2005.

[10] J.M. Borwein and D. M. Zhuang, Verifiable necessary and sufficient conditions for openness and regularity of set-valued and single-valued maps, f. Math. Anal. Appl. 134 (1988), 441-459, doi:10.1016/0022-247x(88)90034-0.

[11] N.H. Chieu, J.C. Yao, and N.D. Yen, Relationships between Robinson metric regularity and Lipschitz-like behavior of implicit multifunctions, Nonlinear Anal. 72 (2010), 3594-3601, doi: 10.1016/j.na.2009.12.039.

[12] C. L. Chiriac, Convergence of the proximal point algorithm variational inequalities with regular mappings, An. Univ. Oradea Fasc. Mat. 17 (2010), 65-69.

[13] T. D. Chuong, Metric regularity of a positive order for generalized equations, Appl. Anal. 94 (2015), 1270-1287, doi:10.1080/00036811.2014.930821.

[14] T.D. Chuong and D. S. Kim, Hölder-like property and metric regularity of a positive-order for implicit multifunctions, Math. Oper. Res. 41 (2016), 596-611, doi:10.1287/moor.2015.0741.

[15] T.D. Chuong, A.Y. Kruger, and J. C. Yao, Calmness of efficient solution maps in parametric vector optimization, f. Global Optim. 51 (2011), 677-688, doi:10.1007/s10898-011-9651-z.

[16] R. Cibulka, J. Preininger, and T. Roubal, On uniform regularity and strong regularity, Optimization 68 (2019), 549-577, doi:10.1080/02331934.2018.1547383.

[17] F. H. Clarke, Optimization and Nonsmooth Analysis, John Wiley \& Sons Inc., New York, 1983.

[18] N.D. Cuong and A. Y. Kruger, Primal Necessary Characterizations of Transversality Properties, Positivity 25 (2021), 531-558, doi:10.1007/s11117-020-00775-5.

[19] A. L. Dontchev, The Graves theorem revisited, f. Convex Anal. 3 (1996), 45-53.

[20] A. L. Dontchev, A.S. Lewis, and R. T. Rockafellar, The radius of metric regularity, Trans. Amer. Math. Soc. 355 (2003), 493-517.

[21] A. L. Dontchev, M. Quincampoix, and N. Zlateva, Aubin criterion for metric regularity, f. Convex Anal. 13 (2006), 281-297.

[22] A. L. Dontchev and R. T. Rockafellar, Implicit Functions and Solution Mappings. A View from Variational Analysis, Springer Series in Operations Research and Financial Engineering, Springer, New York, 2 edition, 2014, doi:10.1007/978-1-4939-1037-3.

[23] A. L. Dontchev and V. M. Veliov, Metric regularity under approximations, Control Cybernet. 38 (2009), 1283-1303.

[24] M. Fabian, Subdifferentiability and trustworthiness in the light of a new variational principle of Borwein and Preiss, Acta Univ. Carolinae 30 (1989), 51-56.

[25] H. Gfrerer and J. V. Outrata, On Lipschitzian properties of implicit multifunctions, SIAM 7. Optim. 26 (2016), 216o-2189, doi:10.1137/15m1052299. 
[26] L. M. Graves, Some mapping theorems, Duke Math. f. 17 (1950), 111-114.

[27] R. Hesse and D. R. Luke, Nonconvex notions of regularity and convergence of fundamental algorithms for feasibility problems, SIAM 7. Optim. 23 (2013), 2397-2419, doi:10.1137/120902653.

[28] N. Q. Huy, D.S. Kim, and K. V. Ninh, Stability of implicit multifunctions in Banach spaces, $\mathcal{F}$. Optim. Theory Appl. 155 (2012), 558-571, doi:10.1007/s10957-012-0058-x.

[29] N. Q. Huy and J. C. Yao, Stability of implicit multifunctions in Asplund spaces, Taiwanese 7. Math. 13 (2009), 47-65, doi:10.11650/twjm/1500405272.

[30] A. D. Ioffe, Metric regularity and subdifferential calculus, Russian Math. Surveys 55 (2000), 501-558, doi:10.1070/rm20oovo55no3abehooo292.

[31] A. D. Ioffe, Metric regularity - a survey. Part I. Theory, f. Aust. Math. Soc. 101 (2016), 188-243, doi:10.1017/s1446788715000701.

[32] A. D. Ioffe, Implicit functions: a metric theory, Set-Valued Var. Anal. 25 (2017), 679-699, doi:10. 1007/s11228-017-0417-8.

[33] A.D. Ioffe, Variational Analysis of Regular Mappings. Theory and Applications, Springer Monographs in Mathematics, Springer, 2017, doi:10.1007/978-3-319-64277-2.

[34] A. D. Ioffe and V. M. Tikhomirov, Theory of Extremal Problems, volume 6 of Studies in Mathematics and its Applications, North-Holland Publishing Co., Amsterdam, 1979.

[35] D. Klatte and B. Kummer, Nonsmooth Equations in Optimization. Regularity, Calculus, Methods and Applications, volume 60 of Nonconvex Optimization and its Applications, Kluwer Academic Publishers, Dordrecht, 2002.

[36] A. Y. Kruger, A covering theorem for set-valued mappings, Optimization 19 (1988), 763-78o, doi: 10.1080/02331938808843391.

[37] A. Y. Kruger, On Fréchet subdifferentials, f. Math. Sci. (N.Y.) 116 (2003), 3325-3358, doi:10.1023/a: 1023673105317. Optimization and Related Topics, 3.

[38] A.Y. Kruger, Error bounds and metric subregularity, Optimization 64 (2015), 49-79, doi:10.1080/ 02331934.2014.938074.

[39] A. Y. Kruger, D. R. Luke, and N. H. Thao, About subtransversality of collections of sets, Set-Valued Var. Anal. 25 (2017), 701-729, doi:10.1007/s11228-017-0436-5.

[40] Y.S. Ledyaev and Q. J. Zhu, Implicit multifunction theorems, Set-Valued Anal. 7 (1999), 209-238, doi:10.1023/a:1008775413250.

[41] G. M. Lee, N. N. Tam, and N.D. Yen, Normal coderivative for multifunctions and implicit function theorems, F. Math. Anal. Appl. 338 (2008), 11-22, doi:10.1016/j.jmaa.2007.05.001.

[42] G. Li and B. S. Mordukhovich, Hölder metric subregularity with applications to proximal point method, SIAM f. Optim. 22 (2012), 1655-1684, doi:10.1137/12086466o.

[43] D. R. Luke, N.H. Thao, and M.K. Tam, Quantitative convergence analysis of iterated expansive, set-valued mappings, Math. Oper. Res. 43 (2018), 1143-1176, doi:10.1287/moor.2017.0898.

[44] L. A. Lyusternik, On the conditional extrema of functionals, Mat. Sbornik 41 (1934), 390-401. 
[45] B. S. Mordukhovich, Variational Analysis and Generalized Differentiation. I: Basic Theory, volume 330 of Grundlehren der Mathematischen Wissenschaften [Fundamental Principles of Mathematical Sciences], Springer, Berlin, 2006.

[46] B.S. Mordukhovich, Variational Analysis and Generalized Differentiation. II: Applications, volume 331 of Grundlehren der Mathematischen Wissenschaften [Fundamental Principles of Mathematical Sciences], Springer, Berlin, 2006.

[47] H. V. Ngai and M. Théra, Error bounds and implicit multifunction theorem in smooth Banach spaces and applications to optimization, Set-Valued Anal. 12 (2004), 195-223, doi:10.1023/b:svan. ooooo 23396.58424 .98 .

[48] H. V. Ngai and M. Théra, Error bounds in metric spaces and application to the perturbation stability of metric regularity, SIAM F. Optim. 19 (2008), 1-20, doi:10.1137/060675721.

[49] H.V. Ngai, N. H. Tron, and M. Théra, Implicit multifunction theorems in complete metric spaces, Math. Program., Ser. B 139 (2013), 301-326, doi:10.1007/s10107-013-0673-9.

[50] T. T. A. Nghia, A note on implicit multifunction theorems, Optim. Lett. 8 (2014), 329-341, doi: 10.1007/s11590-012-0580-7.

[51] R. R. Phelps, Convex Functions, Monotone Operators and Differentiability, volume 1364 of Lecture Notes in Mathematics, Springer-Verlag, Berlin, 2nd edition, 1993.

[52] S. M. Robinson, Stability theory for systems of inequalities. I. Linear systems, SIAM F. Numer. Anal. 12 (1975), 754-769, doi:10.1137/0712056.

[53] S. M. Robinson, Regularity and stability for convex multivalued functions, Math. Oper. Res. 1 (1976), $130-143$.

[54] S. M. Robinson, Stability theory for systems of inequalities. II. Differentiable nonlinear systems, SIAM 7. Numer. Anal. 13 (1976), 497-513.

[55] R. T. Rockafellar, Directionally Lipschitzian functions and subdifferential calculus, Proc. London Math. Soc. (3) 39 (1979), 331-355.

[56] R. T. Rockafellar, Generalized directional derivatives and subgradients of nonconvex functions, Canad. F. Math. 32 (1980), 257-28o, doi:10.4153/cjm-1980-020-7.

[57] R. T. Rockafellar and R. J. B. Wets, Variational Analysis, Springer, Berlin, 1998.

[58] A. Uderzo, On the quantitative solution stability of parameterized set-valued inclusions, Set-Valued Var. Anal 29 (2021), 425-451, doi:10.1007/s11228-020-00571-Z.

[59] C. Ursescu, Multifunctions with convex closed graph, Czechoslovak Math. f. 25(100) (1975), 438-441.

[6o] N.D. Yen and J.C. Yao, Point-based sufficient conditions for metric regularity of implicit multifunctions, Nonlinear Anal. 70 (2009), 2806-2815, doi:10.1016/j.na.2008.04.005.

[61] N. D. Yen, J. C. Yao, and B. T. Kien, Covering properties at positive-order rates of multifunctions and some related topics, f. Math. Anal. Appl. 338 (2008), 467-478, doi:10.1016/j.jmaa.2007.05.041.

[62] C. Zălinescu, Convex Analysis in General Vector Spaces, World Scientific Publishing Co. Inc., River Edge, NJ, 2002, doi:10.1142/9789812777096.

[63] X. Y. Zheng and K. F. Ng, Metric subregularity and calmness for nonconvex generalized equations in Banach spaces, SIAM f. Optim. 20 (2010), 2119-2136, doi:10.1137/090772174. 